\title{
Conditions That Inhibit the Spontaneous Radiation of Spiral Inertia-Gravity Waves from an Intense Mesoscale Cyclone
}

\author{
David A. Schecter And Michael T. Montgomery \\ Department of Atmospheric Science, Colorado State University, Fort Collins, Colorado
}

(Manuscript received 27 October 2004, in final form 14 April 2005)

\begin{abstract}
The spontaneous radiation of spiral inertia-gravity (IG) waves from monotonic cyclones is reexamined. Such radiation can occur most significantly in a parameter regime that includes strong supercell mesocyclones and hurricanes. First, linear theory is reviewed. In linear theory, a generic deformation of the cyclone excites discrete vortex Rossby (VR) waves. Each VR wave emits a frequency-matched spiral IG wave into the environment. The emission has positive feedback on the VR wave, causing both to grow. However, the VR wave also deposits wave activity into its critical layer at the radius $r_{*}$. If the radial gradient of potential vorticity at $r_{*}$ exceeds a threshold, critical layer absorption suppresses the radiative instability.

On the other hand, numerical simulations of a shallow-water cyclone show that nonlinear changes to the critical layer can revive a damped VR wave and its radiation field after a brief period of decay. For such revival, it suffices that $\Omega_{b} /|\gamma| \geqq 1$. This inequality contains two characteristic frequencies. The denominator $|\gamma|$ is the absolute value of the (negative) growth rate of the damped wave. The numerator $\Omega_{b}$ is the mixing rate of the critical layer, which is proportional to the square root of the initial wave amplitude.

After damping is reversed, the radiative VR wave exhibits undulatory growth. Analysis shows that growth proceeds because radiation steadily removes negative wave activity from the cyclone. Secondary amplitude oscillations are due to back-and-forth exchanges of positive wave activity between the VR wave and its critical layer.
\end{abstract}

\section{Overview}

One important mechanism of gravity wave production in the atmosphere is latent heat release by moist updrafts. Another, connected to mountain waves, is topographic forcing. A third, more subtle mechanism, is unforced radiation from jets and vortices, that is, spontaneous radiation. For example, it has been shown that inertia-gravity (IG) waves can emerge from a jet as a by-product of baroclinic instability (e.g., O'Sullivan and Dunkerton 1995). It has also been shown that IG waves can emanate from the vortical motions of ageostrophic turbulence (e.g., Métais and Herring 1989; Polvani et al. 1994). In this paper, we will revisit the paradigm of spontaneous IG wave radiation from a single vortex.

Recent articles have speculated that IG wave radiation can play an important role in the life cycle of me-

Corresponding author address: Dr. David Schecter, Department of Atmospheric Science, Colorado State University, Foothills Campus, Mail 1371, Fort Collins, CO 80523-1371.

E-mail: schecter@atmos.colostate.edu soscale atmospheric vortices over land or sea. Chimonas and Hauser (1997) proposed that it can weaken supercell mesocyclones, under proper conditions, and thereby reduce the chance that they spawn tornadoes. Similarly, Chow and Chan (2003) argued that IG wave radiation can provide an important sink of angular momentum for hurricanes. In the outer region of a hurricane, it might also trigger the formation of spiral rainbands (e.g., Kurihara 1976; Willoughby et al. 1984; Chow et al. 2002).

In section 2, we will identify the basic parameter regime in which spontaneous IG wave radiation can have a relatively large amplitude and appreciably affect the vortex circulation. We will argue that intense tropical cyclones (TCs), supercell mesocyclones, polar lows, and island wake vortices penetrate this regime. Nonetheless, these vortices may not dramatically radiate. The main purpose of this paper is to find conditions that inhibit the spontaneous radiation of IG waves from an otherwise capable vortex.

We will limit our study to pure spontaneous radiation (PSR) (see Ford 1994a,b; Plougonven and Zeitlin 2002; Schecter and Montgomery 2004). By pure, we mean 
spontaneous radiation that sustains its own growth, as opposed to that which is passively amplified by, say, baroclinic or Kelvin-Helmholtz instabilities. An ideal vortex that is immune to such breakdowns, and symmetric instability, is a monotonic shallow-water cyclone (e.g., Montgomery and Shapiro 1995). We will base our theoretical discussion on this model because of its analytical and computational simplicity.

Linear perturbation theory provides the following analysis of PSR: A generic deformation of the cyclone, perhaps a defect of its creation, projects onto a set of discrete azimuthally propagating Rossby waves. Each vortex Rossby (VR) wave emits a frequency-matched spiral IG wave, of proportional strength, into the environment. The radiation has positive feedback on the VR wave, causing both to grow. However, as it propagates, the VR wave resonantly disturbs potential vorticity $(\mathrm{PV})$ in a critical layer centered at the radius $r_{*}$. The critical layer disturbance can have negative feedback on the VR wave and cause it to decay. That is, critical layer damping can oppose radiative pumping and thereby inhibit PSR.

In sections 3 and 4, we will illustrate and closely examine the competition between radiative pumping and critical layer damping in the context of linear theory (cf. Schecter and Montgomery 2004). From an equation that expresses conservation of wave activity, we will derive a formula [Eq. (63)] for the growth rate $\gamma$ of a discrete VR wave and its coupled radiation field. The formula states that $\gamma$ is the sum of a positive radiation term and a negative critical layer term. The magnitude of the critical layer term increases with the cyclone's radial PV gradient at $r_{*}$. If the gradient exceeds a threshold, the negative critical layer term prevails. As a result, the VR wave and its radiation field decay with time.

Damping will persist if linear theory remains valid forever. On the other hand, nonlinear stirring of the critical layer can destabilize the VR wave by removing the mean PV gradient that opposes radiative pumping (cf. Briggs et al. 1970; Pillai and Gould 1994; Montgomery and Enagonio 1998; Schecter et al. 2000; Balmforth et al. 2001). This nonlinear radiative instability occurs if the initial wave amplitude exceeds a critical value, which we will estimate in section 5 [see Eq. (70)]. In section 6 , we will verify the estimated critical value with numerical simulations.

It is well known that the shallow-water equations are isomorphic with the 2D compressible gas equations, with ratio of specific heats $c_{p} / c_{v}=2$. As a result, our study parallels that of acoustic radiation from a vortex in a compressible fluid (e.g., Lighthill 1952; Broadbent and Moore 1979; Kop'ev and Leont'ev 1983, 1985, 1988;
Kambe 1986; Sozou 1987; Zeitlin 1991; Chan et al. 1993; Howe 2001). Although numerical investigations have distinguished vortices that spontaneously emit sound from those that are quiet (e.g., Chan et al. 1993), to our knowledge, the results were not explained in terms of VR wave dynamics. In particular, the results were not explained as the outcome of a competition between critical layer damping and radiative pumping, as they are here.

This paper also overlaps astrophysical studies of modal stability in compressible, differentially rotating discs, with gravitational centers of attraction (e.g., Papaloizou and Pringle 1987; Shukhman 1991; Papaloizou and Lin 1995; Li et al. 2001). The usual example is a stellar accretion disc. The astrophysics literature explicitly considers the effects of both critical layer stirring and spiral radiation on the growth of linear (e.g., Papaloizou and Pringle 1987) and weakly nonlinear (e.g., Shukhman 1991) modes. In addition, the numerical simulations of Li et al. (2001) provide vivid illustrations of spiral radiation that is produced by Rossby-like waves and mesovortices within compressible discs.

More generally, our discussion of nonlinear PSR connects to the broad study of waves with nonlinear critical layers (for an early review, see Maslowe 1986). Such waves include planetary Rossby waves (e.g., Killworth and McIntyre 1985), shallow-water waves in sheared channel flow (e.g., Balmforth 1999), waves in stellar accretion disks (see above), and collisionless plasma waves (e.g., O'Neil 1965). The list also includes nonradiative VR waves in a 2D Euler vortex (Briggs et al. 1970; Pillai and Gould 1994; Schecter et al. 2000; Balmforth et al. 2001).

The remainder of this paper is organized as follows: section 2 identifies the parameter regime in which an atmospheric vortex can spontaneously radiate IG waves with notable intensity. Sections 3 and 4 outline the linear theory of PSR from a monotonic cyclone in the context of the shallow-water model. Section 5 addresses the limitations of linear theory. Section 6 presents a computational study of nonlinear PSR. Section 7 recapitulates our conclusions.

\section{The potential to spontaneously radiate}

In this section, we examine the likelihood of spontaneous radiation from various atmospheric vortices. To facilitate our discussion, we introduce three pertinent dimensionless parameters:

$$
\mathrm{Ro} \equiv \frac{V}{L_{h} f}, \quad \varepsilon \equiv \frac{V}{L_{h} N}, \quad \mathrm{Fr} \equiv \frac{V}{C_{g}} .
$$

Here $V$ and $L_{h}$ are the characteristic rotational velocity 


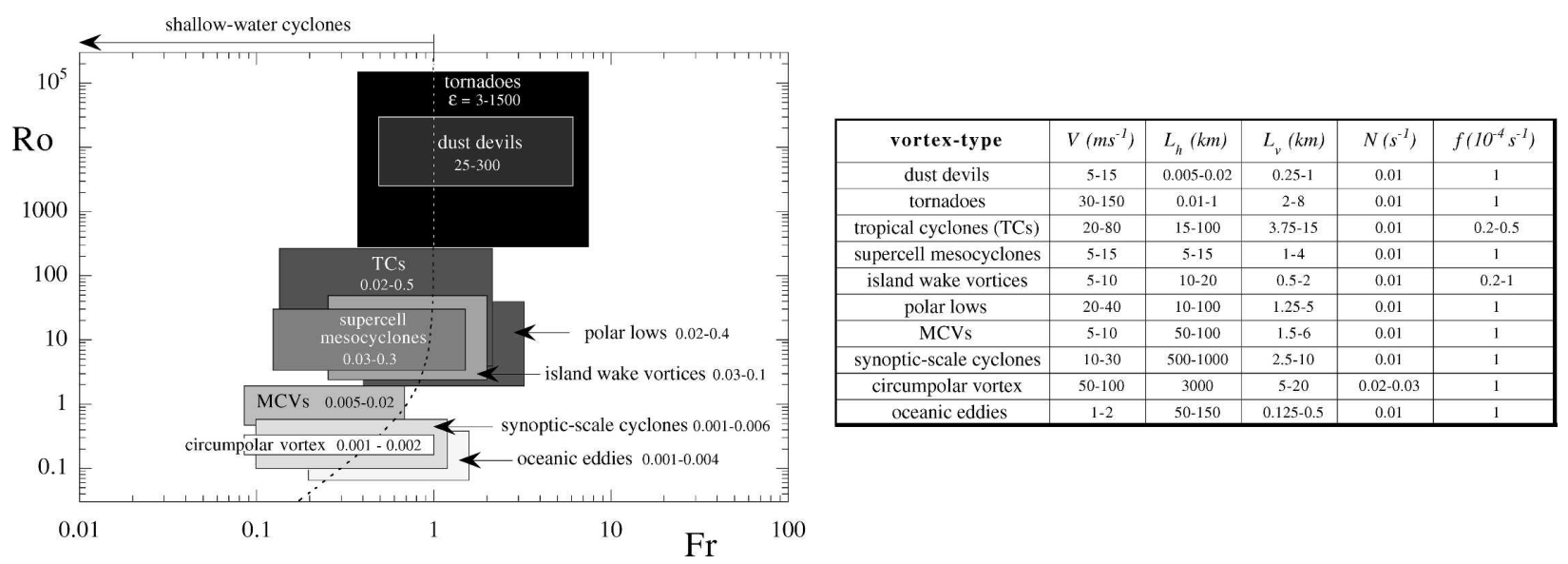

FIG. 1. Estimated parameter regimes for a spectrum of atmospheric vortices and mesoscale oceanic eddies. Balanced shallow-water cyclones reside to the left of the dashed curve. The table on the right provides the characteristic scales that were used to evaluate the ranges of $\mathrm{Ro}, \mathrm{Fr}$, and $\varepsilon$ for each type of vortex.

and horizontal length scale of the vortex. The Rossby number Ro is the vortex rotation frequency over the Coriolis parameter $f$. The $\varepsilon$ parameter is the vortex rotation frequency over the ambient buoyancy frequency $N$. The Froude number Fr is the rotational velocity over the characteristic ambient gravity wave speed $C_{g}$ [see Eq. (5)].

One intuitive requirement for a vortex oscillation, of frequency $\omega$, to generate IG wave radiation is

$$
f \leq \omega \leq N .
$$

Conditions (2) state that radiation can occur only if $\omega$ is within the bounded spectrum of ambient IG waves (cf. Chimonas and Hauser 1997; Ford 1994a,b). Assuming a vortex-scale disturbance, $\omega$ is likely of order $V / L_{h}$, in which case conditions (2) become

$$
\text { Ro } \gtrsim 1 \text { and } \varepsilon \lesssim 1 \text {. }
$$

Here, as usual, the symbols $\gtrsim$ and $\lesssim$ mean "greater than or similar to" and "less than or similar to," respectively. It is perhaps worth noting that violation of (3) does not prohibit exponentially weak emissions (e.g., Vanneste and Yavneh 2004).

Even if the above inequalities are satisfied, the resulting radiation may hardly affect the vortex dynamics. Current asymptotic theories $(\mathrm{Fr} \ll 1)$ suggest that spontaneous IG wave radiation changes the vortex structure at a rate of order $\mathrm{Fr}^{2 n} V / L_{h}$, in which the positive integer $n$ is the dominant azimuthal wavenumber of the vortex asymmetry (e.g., Ford 1994a,b; Ford et al. 2000; Plougonven and Zeitlin 2002). Accordingly, appreciable effects can occur on a rotation time scale only if

$$
\operatorname{Fr} \gtrsim 1 \text {. }
$$

Examples in Schecter and Montgomery (2004) confirm that vortex-scale radiative instabilities can occur rapidly in this parameter regime.

Figure 1 displays our estimates of the dimensionless parameters of different atmospheric vortices, and mesoscale oceanic eddies. The parameter ranges for each case reflect variations of $V, L_{h}, f, N$, and $C_{g}$. The values of $N$ (for atmospheric vortices) correspond to unsaturated environments, that is, approximately dry radiation zones. To estimate the variation of $C_{g}$, we used

$$
C_{g} \sim N L_{v}
$$

in which $L_{v}$ is between one and one-fourth of the maximal vertical length scale of the vortex. Lower values of $N$, which would apply to partially saturated environments, or of $L_{v}$ would decrease $C_{g}$ and therefore increase Fr.

The diagram indicates that, due to small values of Ro, one should not expect vortex-scale vibrations to generate IG wave radiation from oceanic eddies, the circumpolar stratospheric vortex, synoptic-scale cyclones, or mesoscale convective vortices (MCVs). In addition, due to large values of $\varepsilon$, typical oscillations of tornadoes and dust devils should not produce IG wave radiation. We must note, however, that tornadoes can have Mach numbers as large as one-half and emit detectable levels of analogous acoustic radiation (e.g., Abdullah 1966; Bedard 2005).

On the other hand, our estimates suggest that TCs, supercell mesocyclones, polar lows, and island wake vortices can penetrate the parameter regime in which spontaneous IG wave radiation is dynamically relevant. That is, we assert that such vortices can satisfy conditions (3) and (4).

The diagram also shows the parameter space that a 
shallow-water cyclone can access. Inspection of gradient balance [Eq. (14)] suggests that the Froude number of a shallow-water cyclone, with a distinct radial length scale, must satisfy

$$
\mathrm{Fr} \lesssim \frac{1}{\sqrt{1+\mathrm{Ro}^{-1}}}
$$

in order to ensure a nonnegative central surface height. Here, Fr is $V$ over $C_{g}=\sqrt{g h_{\infty}}$, in which $g$ is gravitational acceleration and $h_{\infty}$ is the ambient surface height. The parameter space defined by condition (6) is to the left of the dashed curve in Fig. 1. Notably, Fr is always less than unity. This suggests that spontaneous IG wave radiation might have a weaker effect on shallow-water cyclones than on some atmospheric vortices.

Another corollary of condition (6) is that the intrinsic Rossby deformation radius (Shapiro and Montgomery 1993) of a shallow-water cyclone,

$$
L_{R} \equiv L_{h} \frac{\text { Ro }}{\operatorname{Fr}(1+2 \mathrm{Ro})},
$$

is of order $L_{h}$ or greater in the radiative parameter regime $(R o>1)$. Figure 1 suggests that potentially radiative atmospheric vortices, such as polar lows and TCs, can have smaller values of $L_{R}$, owing to larger values of Fr. The dynamical importance of this parameter is briefly addressed in section 7 .

\section{Pure spontaneous radiation}

In this section, we will briefly analyze the exponentially growing eigenmodes that account for PSR from a monotonic shallow-water cyclone. We will show that each such eigenmode consists of a core VR wave coupled to an outer spiral IG wave. In addition, we will show that smoothing the PV profile of the cyclone can negate the radiative instability.

\section{a. Shallow-water model on the f plane}

The shallow-water equations constitute the simplest model of geophysical flow that accounts for the interaction of vortical motion with IG waves. We may write these equations in compact form,

$$
\begin{aligned}
\partial_{t} \mathbf{u}+\boldsymbol{\eta} \times \mathbf{u}+\nabla\left(\phi+\frac{\mathbf{u}^{2}}{2}\right) & =0, \\
\partial_{t} \phi+\nabla \cdot \phi \mathbf{u} & =0 .
\end{aligned}
$$

Above, $\mathbf{u}$ is the horizontal velocity, $\phi$ is the geopotential ( $g$ times free surface height), $t$ is time, and $\boldsymbol{\nabla}$ is the horizontal gradient operator. We have also introduced the absolute vorticity vector,

$$
\boldsymbol{\eta} \equiv \boldsymbol{\nabla} \times \mathbf{u}+f \hat{\mathbf{z}},
$$

in which $\hat{\mathbf{z}}$ is the vertical unit vector.
It is well known that Eqs. (8) and (9) conserve PV (q) along material trajectories. That is,

$$
\partial_{t} q+\mathbf{u} \cdot \nabla q=0
$$

in which

$$
q \equiv \frac{\hat{\mathbf{z}} \cdot \boldsymbol{\eta}}{\phi} .
$$

The PV Eq. (11) will play an important role in our analysis of critical layer damping.

In what follows, we will use a polar coordinate system, in which $r$ and $\varphi$ denote the radius and azimuth. In addition, we will let $u$ and $v$ denote the radial and azimuthal components of $\mathbf{u}$. Overbars and primes will mark equilibrium and perturbation fields, respectively.

\section{b. Monotonic cyclones}

The shallow-water equations, of course, support cyclonic equilibria. An equilibrated cyclone is characterized by its azimuthal velocity profile $\bar{v}(r)$. Related variables include the following:

$$
\begin{array}{ll}
\bar{\Omega}(r) \equiv \bar{v} / r, & \bar{\zeta}(r) \equiv r^{-1} d(r \bar{v}) / d r, \\
\bar{\eta}(r) \equiv \bar{\zeta}+f, & \bar{\xi}(r) \equiv 2 \bar{\Omega}+f .
\end{array}
$$

Here, $\bar{\Omega}$ is the angular rotation frequency, $\bar{\zeta}$ is the relative vorticity, $\bar{\eta}$ is the absolute vorticity [cf. Eq. (10)], and $\bar{\xi}$ is the modified Coriolis parameter. The geopotential satisfies the gradient balance equation,

$$
\frac{d \bar{\phi}}{d r}=\frac{\bar{v}^{2}}{r}+f \bar{v} .
$$

To facilitate numerical simulations, we will restrict our attention to compact cyclones, for which $\bar{v}$ is zero and $\bar{\phi}$ is constant for $r$ greater than some radius $r_{b}$. In addition, we will assume that the unperturbed potential vorticity $\bar{q}(r)=\bar{\eta} / \bar{\phi}$ decreases monotonically with radius for $r<r_{b}$.

Specifically, we will consider two types of cyclones. The first is a Gaussian cyclone,

$$
\bar{q}=\frac{b_{1}}{f r_{o}^{2}}\left(1+b_{2} e^{-r^{2} / r_{o}^{2}}\right), r<r_{b}
$$

and the second is a hyperbolic cyclone,

$$
\bar{q}=\frac{b_{1}}{f r_{o}^{2}}\left\{1+\frac{b_{2}}{2}\left[1-\tanh \left(\frac{r-r_{o}}{r_{o} \Delta}\right)\right]\right\}, r<r_{b} .
$$

In both cases, $r_{o}$ is the core radius of the cyclone, $r_{b}=$ $22 r_{o}, b_{1}=2.25 \times 10^{-4}$, and $b_{2}=400$. The parameters $b_{1}$ and $b_{2}$ were adjusted to obtain relatively high Rossby and Froude numbers, so as to nearly maximize the influence of IG wave radiation on the vortex dynamics. 

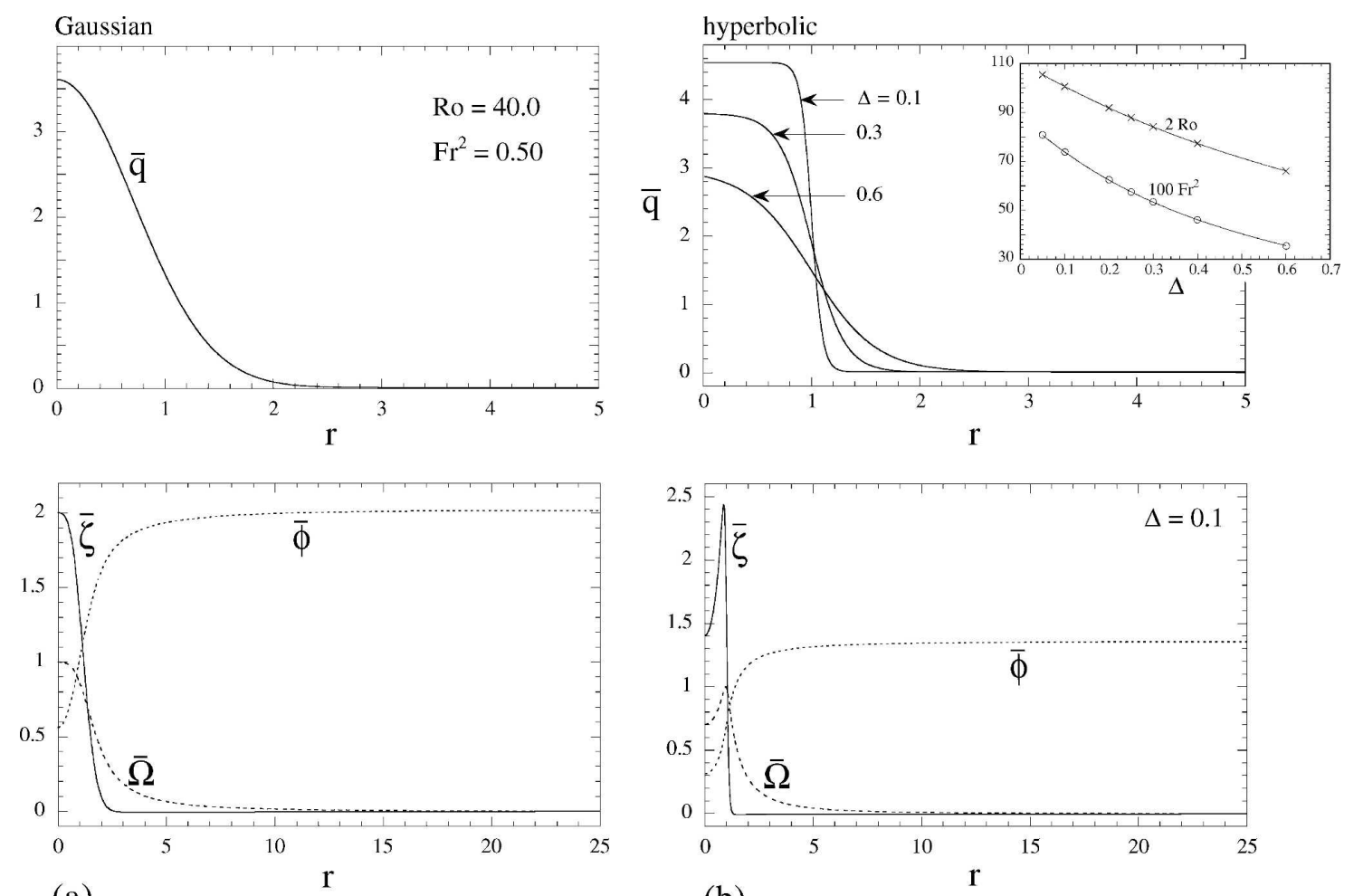

(b)

FIG. 2. Balanced (a) Gaussian and (b) hyperbolic cyclones. All plots are in natural units [see Eq. (17)].

Figure 2 portrays both types of cyclones. All dimensional variables are given in units of natural time and length,

$$
t_{\text {nat }} \equiv \bar{\Omega}_{\text {max }} t \quad \text { and } \quad r_{\text {nat }} \equiv r / r_{o},
$$

in which max indicates the maximum value of the subscripted field. The Rossby and Froude numbers were obtained from the equations

$$
\mathrm{Ro}=\frac{\bar{\Omega}_{\mathrm{max}}}{f} \text { and } \operatorname{Fr}=\frac{r_{o} \bar{\Omega}_{\max }}{\sqrt{\bar{\phi}(\infty)}} .
$$

To compute $\bar{v}$ and $\bar{\phi}$, given $\bar{q}$, we generalized the method of Ford (1994a) to permit finite values of $r_{b}{ }^{1}$

As stated previously, beyond $r_{b}$ we impose the rest condition, $\bar{v}=0$. This leads to a slight jump of PV $\left[\leq 10^{-3} \bar{q}(0)\right]$; specifically,

$$
\left.\bar{q}\right|_{r_{b}^{-}} ^{r_{b}^{+}}=-\frac{1}{\bar{\phi}(\infty)} \frac{d \bar{v}}{d r_{b}^{-}} .
$$

\footnotetext{
${ }^{1}$ In addition, we generalized the method to find $\bar{v}$ and $\bar{\phi}$ on a complex radial contour, which is required to compute quasimode frequencies (see section $3 \mathrm{e}$ ).
}

Since $r_{b} \gg r_{o}$, the jump has negligible consequence on the vortex dynamics, for most considerations.

\section{c. Eigenmodes}

The eigenmodes of a shallow-water cyclone are traveling wave perturbations of the form

$$
\phi^{\prime}(r, \varphi, t)=a \Phi(r) e^{i(n \varphi-\omega t)}+\text { c.c. }
$$

and likewise for the velocity fields. Here $a$ is a dimensionless amplitude, $n$ is the azimuthal wavenumber, c.c. is short for complex conjugate, and $\omega$ is a complex frequency. Explicitly,

$$
\omega \equiv \omega_{R}+i \gamma,
$$

in which both $\omega_{R}$ and $\gamma$ are real.

Upon substituting a traveling wave disturbance (20) into the linearized shallow-water equations (appendix A) and simplifying, we obtain

$$
\begin{gathered}
{\left[\frac{1}{r} \frac{d}{d r} \frac{r \bar{\phi}}{\bar{\eta} \bar{\xi}-\sigma^{2}} \frac{d}{d r}-\frac{n}{r \sigma} \frac{d}{d r}\left(\frac{\bar{\phi} \bar{\xi}}{\bar{\eta} \bar{\xi}-\sigma^{2}}\right)-\frac{n^{2} \bar{\phi}}{r^{2}\left(\bar{\eta} \bar{\xi}-\sigma^{2}\right)}-1\right] \Phi} \\
=0,
\end{gathered}
$$


eigenmode

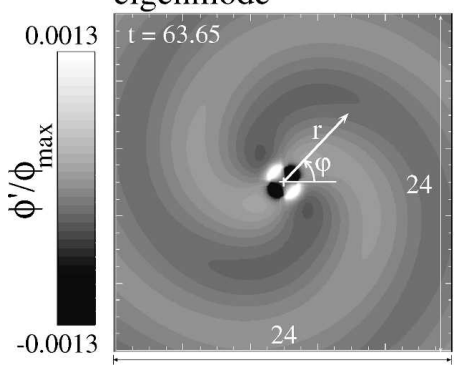

quasimode
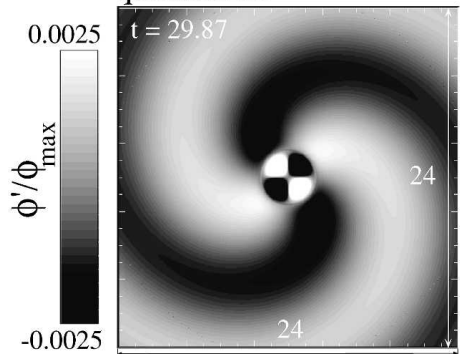
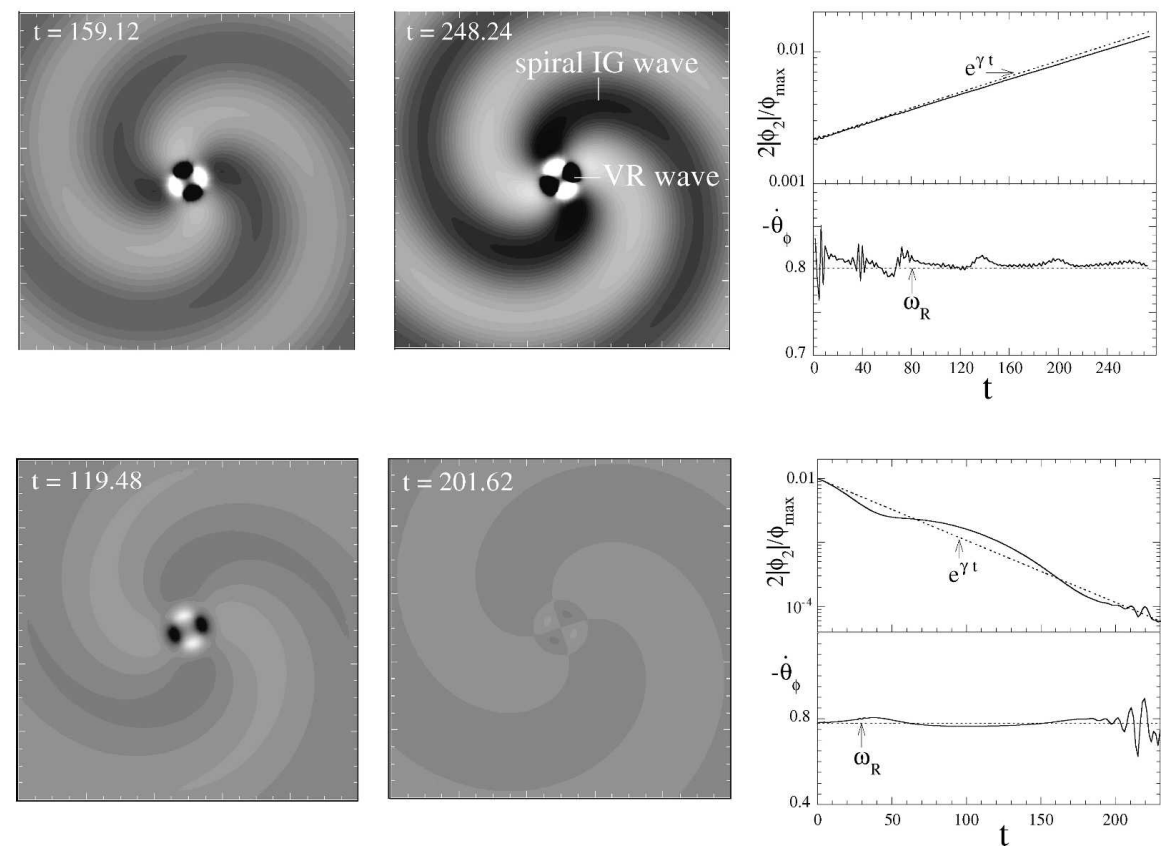

FIG. 3. Simulations of (top) sustained and (bottom) damped spontaneous radiation from hyperbolic cyclones with $\Delta=0.1$ and $\Delta=$ 0.3 , respectively. The contour plots show the geopotential perturbation $\phi^{\prime}$. In both cases, a core VR wave excites an outwardpropagating spiral IG wave in the environment. Section 4 explains why the radiation intensifies in the top case but decays in the bottom case. The graphs to the right show the VR wave amplitude and oscillation frequency vs time. In both cases, the evolution agrees with linear eigenmode theory (dashed curves), generalized to include quasimodes (see section 3e). The wave measurements were obtained from the $n=2$ complex Fourier coefficient of the geopotential perturbation, $\phi_{2}(r, t) \equiv\left|\phi_{2}\right| e^{i \theta_{\phi}}$, evaluated at (top) $r=1.03 r_{o}$ and (bottom) $r=r_{o}$. Times and lengths are in natural units; $\phi_{\max }$ is the initial maximum value of the geopotential (approximately the ambient geopotential).

in which

$$
\sigma(r) \equiv \omega-n \bar{\Omega}
$$

is the complex Doppler-shifted wave frequency. On occasion, we will use $\sigma_{R}$ to represent the real part of $\sigma$.

It is of interest to consider the asymptotic solutions of $\Phi$ near the origin and in the radiation zone, $r>r_{b}$. The regular solution near the origin is given by

$$
\Phi \propto r^{n}, \quad r \rightarrow 0 .
$$

In the radiation zone, $\Phi$ is a Hankel function of the first or second kind. If we require that the wave propagates radially outward, we obtain

$$
\Phi \propto H_{n}^{(1)}(\kappa), \quad r>r_{b},
$$

in which

$$
\kappa \equiv \sqrt{\frac{\omega^{2}-f^{2}}{\bar{\phi}(\infty)}} r .
$$

By convention, the argument of the square root is between $-\pi / 2$ and $+\pi / 2$.

Equations (24) and (25) provide mixed boundary conditions, that is, relations between $\Phi$ and $d \Phi / d r$ at points near the origin and in the radiation zone. The values of $\omega$ that permit regular solutions to Eq. (22) with these boundary conditions are obtained numerically by a standard shooting technique. Appendix B provides formulas for relating the eigenfunction $\Phi$ to the velocity and PV wavefunctions.

\section{d. Radiative instability}

Figure 3 illustrates the responses of two hyperbolic cyclones to slight elliptical deformations. We first consider the top row, which is from a numerical simulation of the nonlinear shallow-water equations (see section 6a).

After a brief transition period, the most unstable $n=$ 2 eigenmode dominates the perturbation. In the outer region, where $d \bar{q} / d r$ is negligible, the eigenmode is an outward propagating spiral IG wave. It is locally prograde $\left(\sigma_{R}>0\right)$ and fast $\left(\sigma_{R}>\bar{\xi}\right)$. According to Eq. (25), its asymptotic radial wavelength is

$$
l_{\mathrm{rad}} \approx 2 \pi \sqrt{\frac{\bar{\phi}(\infty)}{\omega_{R}^{2}-f^{2}}}=\frac{2 \pi r_{o}}{\operatorname{Fr} \sqrt{\left(\omega_{R} / \bar{\Omega}_{\mathrm{max}}\right)^{2}-\mathrm{Ro}^{-2}}} .
$$


In contrast, the inner part of the eigenmode is a VR wave. It is locally retrograde $\left(\sigma_{R}<0\right)$ and slow $\left(\left|\sigma_{R}\right|<\right.$ $\bar{\xi})$. In addition, its polarization closely resembles that of Kelvin's quintessential VR wave (Kelvin 1880). Hence, the radiative instability involves a resonant coupling between inner and outer VR and IG waves.

\section{e. Quasimodes}

Figure 4 shows that increasing the smoothness parameter $\Delta$ of the hyperbolic cyclone decreases the growth rate $\gamma$ of its $n=2$ radiative eigenmode. Moreover, increasing $\Delta$ above a threshold quenches the radiative instability. Before explaining the physics of this transition (see section 4d), there is an important technical issue to address.

As the growth rate of the radiative mode becomes negative, it transforms from an eigenmode into a quasimode. Quasimodes are not exact solutions to the eigenmode problem. For example, the PV disturbance of a quasimode decays with time over the bulk of the cyclone, but grows with time in a thin critical layer (e.g., Schecter et al. 2000, 2002). In contrast, an eigenmode would decay everywhere. ${ }^{2}$

The aforementioned critical layer is centered at $r_{*}$, where the rotation frequency of the cyclone equals the angular phase velocity of the mode; that is,

$$
\bar{\Omega}\left(r_{*}\right)=\omega_{R} / n .
$$

Although Eq. (28) can have multiple solutions, we will only consider cases for which there is a unique critical radius $r_{*}$ outside the vortex core. The radial width of the critical layer (in linear theory) is of order

$$
l_{\gamma} \equiv\left|\frac{\gamma}{n d \bar{\Omega} / d r_{*}}\right|,
$$

in which $d / d r_{*}$ is the radial derivative evaluated at $r_{*}$.

A formal Laplace transform solution to the initial value problem leads to a simple procedure for computing the complex frequency $\omega$ of a quasimode. The procedure has been derived for the quasimodes of 2D Euler vortices (Briggs et al. 1970; Corngold 1995; Spencer and Rasband 1997) and for the quasimodes of 3D barotropic cyclones in density stratified fluids (Schecter et al. 2002; Schecter and Montgomery 2004). Below, we

\footnotetext{
${ }^{2}$ Note also that the asymptotic wavefunction [Eq. (25)] of a quasimode ultimately increases with $r$, since $\gamma<0$. We assert that such behavior is physical, since the outer radiation field travels at a finite radial velocity, and is generated by a central source (the VR wave) that decays exponentially with time.
}

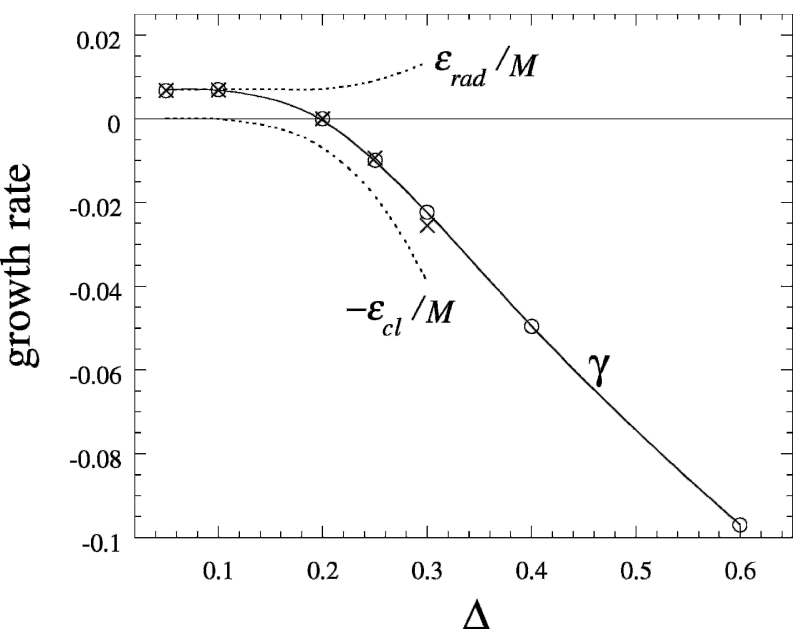

FIG. 4. Growth rate $\gamma$ of the $n=2$ radiative VR wave vs the smoothness parameter $\Delta$ of a hyperbolic cyclone. Numerical solutions to the eigenmode/quasimode problem $(\bigcirc)$ are compared to analytical growth rates $(\times)$, where applicable. The dashed curves show the radiative pumping and critical layer damping contributions, $\epsilon_{\mathrm{rad}} / M$ and $-\epsilon_{\mathrm{cl}} / M$, to the analytical growth rates [see Eq. (63)]. A growth rate of 0.1 in natural units corresponds to an $e$-folding time of 1.59 vortex rotation periods.

describe the analogous procedure for a shallow-water cyclone, without derivation.

To find the complex quasimode frequency, we must solve Eqs. (22)-(25) for $\omega$ along a complex radial contour. That is, we must replace $r$ with the complex variable $\rho(r) \equiv r+i s(r)$, and $d / d r$ with $d / d \rho$. The endpoints of the contour are fixed on the real axis at $r=0^{+}$and at any $r>r_{b}$. Moreover, assuming that $d \bar{\Omega} / d r_{*}<0$, the contour must arc over the complex critical radius $\rho_{*}$, defined by

$$
\bar{\Omega}\left(\rho_{*}\right) \equiv \omega / n
$$

If $\gamma$ is small, then

$$
\rho_{*} \approx r_{*}+i l_{\gamma}
$$

In this paper, where it is necessary to evaluate a quasimode wavefunction, we let $s(r)=0$ except in the interval $r_{*} \pm 2 l_{\gamma}$. There, $s$ increases to a peak value of $2 l_{\gamma}$ and then returns to zero. We estimate that the velocity and geopotential wavefunctions obtained along this special contour have an error of order $l_{\gamma} / r_{*}$ in the critical layer. The PV wavefunction, although accurate over the bulk of the cyclone, is meaningless in the critical layer.

The bottom row of Fig. 3 illustrates the behavior of a quasimode in a hyperbolic cyclone, with $\Delta=0.3$. In this case, the data were obtained from a numerical integration of the linearized shallow-water equations (appen- 
dix A). A sponge ring was applied at large radii to absorb outward-propagating waves. The initial conditions were given by the $n=2$ quasimode wavefunction, computed along $\rho(r)$. The pressure anomaly closely resembles a pure eigenmode, but its time dependence deviates slightly from exponential decay. The deviation is an artifact of the PV blemish seeded in the critical layer of the wavefunction.

\section{Radiative pumping versus critical layer damping}

In this section, we will explain why a VR wave is compelled to grow upon emitting a frequency matched IG wave into the environment. In addition, we will explain why resonant stirring of PV in the critical layer (at $\left.r_{*}\right)$ counters such growth. Importantly, we will find that critical layer damping prevails over radiative pumping if the magnitude of $d \bar{q} / d r_{*}$ exceeds a finite threshold. Finally, we will reexamine the stabilization of hyperbolic cyclones, by smoothing, in the context of these results.

\section{a. Angular pseudomomentum}

Our analysis is based on the equation that governs angular pseudomomentum (e.g., Guinn and Schubert 1993). The angular pseudomomentum of a disturbance in a shallow-water cyclone is given by

$$
\mathcal{L}(r, \varphi, t) \equiv \phi \Upsilon+r v^{\prime} \phi^{\prime},
$$

in which

$$
\Upsilon(r, q) \equiv \int_{\bar{q}(r)}^{q} d \tilde{q}[m(\tilde{q})-m(\bar{q})]
$$

and

$$
m(q) \equiv \int_{0}^{r(q)} d \tilde{r} \tilde{r} \bar{\phi}(\tilde{r})
$$

Here $r(q)$ is the inverse of the function $\bar{q}(r)$. To lowest order in the perturbation amplitude, the angular pseudomomentum reduces to

$$
\mathcal{L} \rightarrow \frac{r}{2} \frac{\bar{\phi}^{2}}{d \bar{q} / d r}\left(q^{\prime}\right)^{2}+r v^{\prime} \phi^{\prime} .
$$

That is, to lowest order, $\Upsilon$ is proportional to the squared PV perturbation. Figure 5 plots the exact structure of $\Upsilon$, in the $r-q$ plane, for a hyperbolic cyclone. The zero contour is the curve $q=\bar{q}(r)$.

In the absence of forcing and dissipation, $\mathcal{L}$ satisfies the equation

$$
\partial_{t} \mathcal{L}+\nabla \cdot \mathbf{F}=0
$$

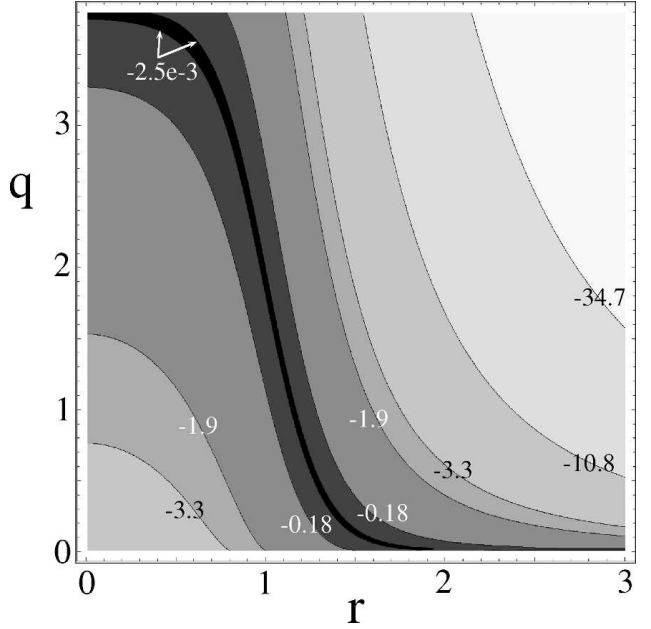

FIG. 5. The $\Upsilon$ function that appears in the definition of angular pseudomomentum $\mathcal{L}$, for the hyperbolic cyclone of simulations H1-H3 $(\Delta=0.3)$. Darker shades indicate smaller magnitudes. The center of the darkest region is the zero contour, $q=\bar{q}(r)$. All numbers are in natural units.

in which

$$
\begin{aligned}
\mathbf{F}(r, \varphi, t) \equiv & \left(u \mathcal{L}+r \bar{\phi} u^{\prime} v^{\prime}\right) \hat{r} \\
& +\left\{v \mathcal{L}+\frac{r \bar{\phi}}{2}\left[\left(v^{\prime}\right)^{2}-\left(u^{\prime}\right)^{2}\right]+\frac{r}{2}\left(\phi^{\prime}\right)^{2}\right\} \hat{\varphi}
\end{aligned}
$$

is the angular pseudomomentum flux vector.

\section{b. Conservation of wave activity}

We define the total wave activity as the integral

$$
A \equiv \int_{0}^{\infty} d r\left(\Lambda_{q}+\Lambda_{v \phi}\right)
$$

in which

$$
\Lambda_{q}(r, t) \equiv-\frac{1}{4 \pi} \int_{0}^{2 \pi} d \varphi r \phi \Upsilon
$$

and

$$
\Lambda_{v \phi}(r, t) \equiv-\frac{1}{4 \pi} \int_{0}^{2 \pi} d \varphi r^{2} v^{\prime} \phi^{\prime} .
$$

The sum, $\Lambda_{\text {tot }}(r, t) \equiv \Lambda_{q}+\Lambda_{v \phi}$, is proportional to the instantaneous azimuthal mean of angular pseudomomentum $\mathcal{L}$ at $r$. The negative constant of proportionality is a convention of this paper.

Suppose that a single VR wave dominates the perturbation in the bulk of the vortex. Then, it proves useful to divide $A$ into bulk wave (b), critical layer (cl), 
and environmental (en) components. That is, let $A=$ $A_{b}+A_{\mathrm{cl}}+A_{\mathrm{en}}$, in which

$$
\begin{aligned}
A_{b}\left(t ; r_{v}, r_{*}, \delta r_{*}\right) & \equiv \int_{0}^{r_{v}} d r \Lambda_{\mathrm{tot}}, \\
A_{\mathrm{cl}}\left(t ; r_{*}, \delta r_{*}\right) & \equiv \int_{r_{*}-\delta r_{*}}^{r_{*}+\delta r_{*}} d r \Lambda_{\mathrm{tot}},
\end{aligned}
$$

and

$$
A_{\mathrm{en}}\left(t ; r_{v}\right) \equiv \int_{r_{v}}^{\infty} d r \Lambda_{\mathrm{tot}} .
$$

Here $r_{v}$ is the outer radius of the vortex where, loosely speaking, radiation is emitted into the environment. The symbol $f$ represents an integral that excludes the critical layer, $\left|r-r_{*}\right|<\delta r_{*}$. For now, let it suffice to say that $\delta r_{*} \ll r_{*}$ and $r_{v}>r_{*}+\delta r_{*}$.

Integrating Eq. (36) over space and dividing through by $-4 \pi$, we obtain the conservation law

$$
\frac{d}{d t} A_{b}=-\frac{d}{d t} A_{\mathrm{en}}-\frac{d}{d t} A_{\mathrm{cl}}
$$

Equation (44) states that bulk wave activity must decay/ grow to compensate gains/losses by the environment and by the critical layer. ${ }^{3}$

Alternative forms of Eq. (44) that we will use in this paper include

$$
\frac{d}{d t} A_{b}=S\left(r_{v}, t\right)-\frac{d}{d t} A_{\mathrm{cl}}
$$

and

$\frac{d}{d t} A_{b}=S\left(r_{v}, t\right)-\left[S\left(r_{*}+\delta r_{*}, t\right)-S\left(r_{*}-\delta r_{*}, t\right)\right]$,

in which

$$
S(r, t) \equiv \frac{1}{4 \pi} \int_{0}^{2 \pi} d \varphi r \hat{\mathbf{r}} \cdot \mathbf{F} .
$$

Equations (45) and (46) are obtained from Eq. (44) by the substitutions

$$
\frac{d}{d t} A_{\mathrm{en}} \rightarrow-S\left(r_{v}, t\right)
$$

\footnotetext{
${ }^{3}$ In case $r \mathbf{F}$ does not vanish at infinity, we redefine $d A_{\mathrm{en}} / d t$ to be the rate at which wave activity enters the environment only from the vortex [Eq. (48)]. This would differ from the time derivative of the improper integral in Eq. (43).
}

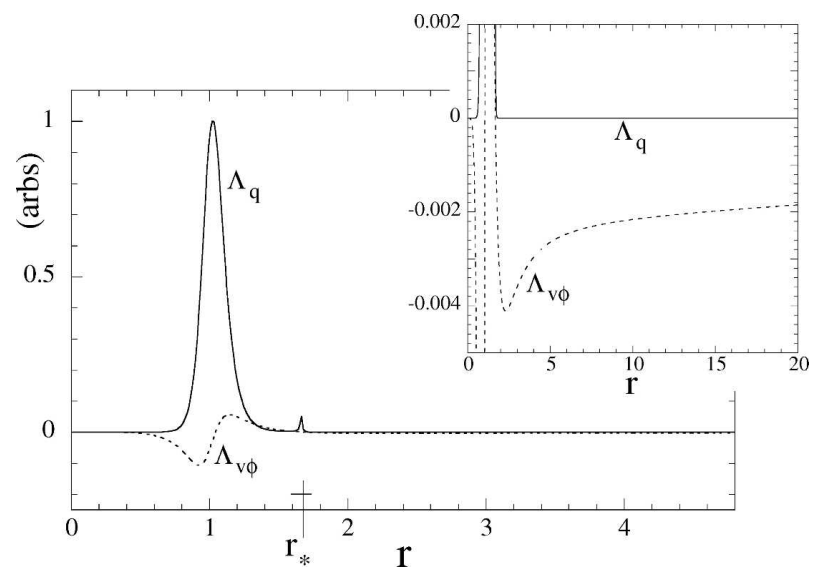

FIG. 6. Wave activity densities for the $n=2$ radiative mode of a hyperbolic cyclone, with $\Delta=0.1$. The solid curve is the quadratic approximation of $\Lambda_{q}$, whereas the dashed curve is $\Lambda_{v \phi}$. Both are scaled by the same arbitrary factor. Their sum, the total wave activity density, is mostly positive for the inner VR wave but is negative for the outer IG wave (see inset). The horizontal bar on the cross-hair at the base of the plot indicates the region that the $M$ integral [Eq. (53)] is supposed to exclude $\left(\left|r-r_{*}\right|<10 l_{\gamma}\right)$. Radius $r$ is in natural units.

and

$$
\frac{d}{d t} A_{\mathrm{cl}} \rightarrow S\left(r_{*}+\delta r_{*}, t\right)-S\left(r_{*}-\delta r_{*}, t\right)
$$

The above identities follow directly from the appropriate spatial integrals of Eq. (36).

One may view the growth of a radiative mode (e.g., Fig. 3, top row) to be a consequence of conservation of wave activity. Figure 6 is a snapshot of the wave activity densities, $\Lambda_{q}$ and $\Lambda_{v \phi}$, of the $n=2$ radiative mode of a hyperbolic cyclone. Over the bulk of the cyclone the positive PV component $\Lambda_{q}$ dominates; that is, the VR wave has positive wave activity. In contrast, the negative $\Lambda_{v \phi}$ component dominates the outer IG wave activity. This suggests that IG wave radiation involves the ejection of negative wave activity into the environment. To conserve total wave activity, it would seem that the bulk VR wave must grow. ${ }^{4}$ On the other hand, the following subsection will show that the growth is slowed, and possibly reversed, by transfer of positive wave activity from the bulk VR wave into the critical layer.

\footnotetext{
${ }^{4}$ Similarly, Kop'ev and Leont'ev $(1983,1985,1988)$ used an energy argument to explain spontaneous acoustic radiation. From their perspective, a negative energy VR wave grows by the ejection of a positive energy sound wave.
} 


\section{c. The growth rate of a radiative $V R$ wave: Linear theory}

Our next goal is to convert Eq. (45) into an amplitude equation for a radiative VR wave. For this analysis, we treat the wave as a perturbation of the form

$$
\left[\begin{array}{l}
u^{\prime}(r, \varphi, t) \\
v^{\prime}(r, \varphi, t) \\
\phi^{\prime}(r, \varphi, t)
\end{array}\right] \approx\left[\begin{array}{l}
a(t) e^{i\left(n \varphi-\omega_{R} t\right)} U(r)+\text { c.c. } \\
a(t) e^{i\left(n \varphi-\omega_{R} t\right)} V(r)+\text { c.c. } \\
a(t) e^{i\left(n \varphi-\omega_{R} t\right)} \Phi(r)+\text { c.c. }
\end{array}\right] .
$$

Here, in contrast to Eq. (20), the amplitude $a$ is an undetermined function of time with slowly varying phase, and the frequency $\omega_{R}$ in the exponential is real.

In the critical layer we permit a small correction to the above "single-wave" approximation [Eq. (50)]. To emphasize its potential deviance, we separate the critical layer disturbance from the bulk disturbance. For example, the PV perturbation becomes

$$
q^{\prime}(r, \varphi, t)= \begin{cases}a(t) e^{i\left(n \varphi-\omega_{R} t\right)} Q(r)+\text { c.c., } & \left|r-r_{*}\right|>\delta r_{*}, \\ \hat{q}_{\mathrm{cl}}(r, t) e^{i n \varphi}+\text { c.c., } & \left|r-r_{*}\right|<\delta r_{*} .\end{cases}
$$

In what follows, we will assume that the linearized shallow-water equations (appendix A) govern the evolution of all parts of the disturbance. Accordingly, we will evaluate all wave activity integrals and fluxes in Eq. (45) to lowest order (second order) in the perturbation amplitude. In addition, we will let $\delta r_{*}=c l_{\gamma}$ so that the interval $\left|r-r_{*}\right|<\delta r_{*}$ covers the entire linear critical layer. For the numerical computations in section $4 \mathrm{~d}$, we will generally let $c=10$.

Substituting the single-wave approximation [Eq. (50)] into the right-hand side of Eq. (41) and differentiating with respect to time, we obtain

$$
\frac{d}{d t} A_{b}=M|a| \frac{d|a|}{d t}
$$

in which

$$
M \equiv \int_{0}^{r_{v}} d r\left\{\frac{r^{2} \bar{\phi}^{2}|Q|^{2}}{-d \bar{q} / d r}-2 r^{2} \Re\left[V \Phi^{*}\right]\right\} .
$$

Here, $\Re[$. . .] denotes the real part of the quantity in square brackets, and the superscript asterisk is the complex conjugate operator.

We may also express $S\left(r_{v}, t\right)$ as a function of wave amplitude. Substituting Eq. (50) into the right-hand side of Eq. (47), and evaluating at $r_{v}$ yields

$$
S\left(r_{v}, t\right)=\epsilon_{\text {rad }}|a|^{2},
$$

in which

$$
\epsilon_{\mathrm{rad}} \equiv\left\{r^{2} \bar{\phi} \Re\left[U V^{*}\right]\right\}_{r_{v}}
$$

Now, the rate of change of wave activity in the critical layer can be written

$$
\begin{aligned}
\frac{d}{d t} A_{\mathrm{cl}}= & -\frac{1}{2} \int_{r_{*}-c l_{\gamma}}^{r_{*}+c l_{\gamma}} d r \\
& \times\left[\frac{r^{2} \bar{\phi}^{2}}{d \bar{q} / d r} \partial_{t}\left(\hat{q}_{\mathrm{cl}} \hat{q}_{\mathrm{c} 1}^{*}\right)+r^{2} \partial_{t}\left(\hat{v}_{\mathrm{cl}} \hat{\phi}_{\mathrm{cl}}^{*}+\text { c.c. }\right)\right] .
\end{aligned}
$$

Let us consider both terms of the integrand separately.

The first term is proportional to $\partial_{t}\left(\hat{q}_{\mathrm{cl}} \hat{q}_{\mathrm{cl}}^{*}\right)$. To evaluate this expression, we solve the linearized PV equation in the critical layer. This equation simplifies to

$$
\partial_{t} \hat{q}_{\mathrm{cl}}+i n \bar{\Omega} \hat{q}_{\mathrm{cl}}=-a U e^{-i \omega_{R^{t}}} \frac{d \bar{q}}{d r},
$$

if we approximate $u^{\prime}$ by its dominant single-wave component. We are justified in doing so provided that $u^{\prime}$ is multiplied by a small parameter $\left(d \bar{q} / d r\right.$ near $\left.r_{*}\right)$.

Equation (57) is identical to that which governs PV in the linear critical layer of a VR wave in a 3D barotropic cyclone. We refer the reader to section 5 of Schecter and Montgomery (2004) for details of its integration and subsequent evaluation of $\partial_{t}\left(\hat{q}_{\mathrm{cl}} \hat{q}_{\mathrm{cl}}^{*}\right)$. Under the assumption that $a$ changes on a time scale much slower than $\omega_{R}^{-1}$, the solution tells us that

$$
\partial_{t}\left(\hat{q}_{\mathrm{cl}} \hat{q}_{\mathrm{cl}}^{*}\right) \rightarrow 2 \pi|U|^{2}\left(\frac{d \bar{q}}{d r}\right)^{2} \frac{\delta\left(r-r_{*}\right)}{n|d \bar{\Omega} / d r|}|a|^{2},
$$

after several oscillation periods. Here $\delta\left(r-r_{*}\right)$ is the Dirac delta function centered at $r_{*}$. The sharply peaked growth of the PV anomaly is a manifestation of waveflow resonance at the critical radius.

We now consider the second term of the integrand. A Frobenius analysis of exponentially growing waves suggests that, in the critical layer, $\hat{v}_{\mathrm{cl}} \hat{\phi}_{\mathrm{cl}}^{*}+$ c.c. diverges logarithmically, at worst, with decreasing $l_{\gamma}$ (cf. Schecter and Montgomery 2004). We may also assume that differentiation with respect to time multiplies this term by a factor that is proportional to $l_{\gamma}$. So, if the growth rate is small, that is, if $l_{\gamma}$ is sufficiently close to zero, it appears safe to use the approximation

$$
\int_{r_{*}-c l_{\gamma}}^{r_{*}+c l_{\gamma}} d r r^{2} \partial_{t}\left(\hat{v}_{\mathrm{cl}} \hat{\phi}_{\mathrm{cl}}^{*}+\text { c.c. }\right) \rightarrow 0 .
$$

Substituting Eqs. (58) and (59) into the right-hand side of Eq. (56) yields

$$
\frac{d A_{\mathrm{cl}}}{d t}=\epsilon_{\mathrm{cl}}|a|^{2},
$$


in which

$$
\epsilon_{\mathrm{cl}} \equiv-\left.\pi \frac{r^{2} \bar{\phi}^{2}|U|^{2} d \bar{q} / d r}{n|d \bar{\Omega} / d r|}\right|_{r_{*}} .
$$

Finally, substituting Eqs. (52), (54), and (60) into Eq. (45) we obtain

$$
\frac{d}{d t}|a|=\gamma|a|
$$

in which

$$
\gamma \equiv \frac{\epsilon_{\mathrm{rad}}-\epsilon_{\mathrm{cl}}}{M}
$$

\section{d. Interpretation and verification of the growth rate formula}

To interpret the analytical growth rate formula [Eq. (63)], we first observe that the $M$ integral is positive if $r_{v}$ is not too large. Figure 6 validates our assertion for the $n=2$ radiative mode of a hyperbolic cyclone. Simply note that the integrand of $M$ is directly proportional to the net-positive bulk wave activity density in this figure.

According to Eq. (63), the growth rate $\gamma$ of a VR wave is the sum of two terms. The first term, $\epsilon_{\mathrm{rad}} / M$, accounts for the feedback of radiation. It is positive if the VR wave excites an outward-propagating spiral IG wave at $r_{v}$. The second term, $-\epsilon_{\mathrm{cl}} / M$, gives the feedback of $\mathrm{PV}$ stirring in the critical layer. It is negative for monotonic cyclones. Moreover, if the magnitude of $d \bar{q} / d r_{*}$ exceeds a threshold, the negative critical layer term prevails. As a result, the VR wave and its radiation field decay with time.

We now return to Fig. 4, which shows the growth rate of a radiative VR wave of a hyperbolic cyclone, with variable smoothness. The $\times$ markers correspond to Eq. (63). To evaluate the right-hand side of this equation, we used the wavefunctions $(U, V, \Phi, Q)$ and critical layer parameters $\left(r_{*}, l_{\gamma}\right)$ of the computed eigenmodes or quasimodes. Evidently, Eq. (63) accurately describes the competition between radiative pumping and critical layer damping, near marginal stability.

Figure 4 also shows how the radiative pumping rate $\left(\epsilon_{\mathrm{rad}} / M\right)$ and critical layer damping rate $\left(\epsilon_{\mathrm{cl}} / M\right)$ vary with the smoothness parameter $\Delta$. For these curves, $\epsilon_{\mathrm{rad}}$ and $M$ were evaluated with $r_{v}=12 r_{o}$. As $\Delta$ increases from 0.05 to 0.3 , the radiative pumping rate increases

\footnotetext{
${ }^{5}$ We have verified that the dashed curves in Fig. 4 are qualitatively the same for any vortex radius in the range $r_{*}+10 l_{\gamma}<r_{v}$ $<48 r_{o}$. We have not examined larger values of $r_{v}$.
}

slightly. However, the PV gradient at $r_{*}$ explosively grows, and the critical layer damping rate increases by orders of magnitude. Consequently, $\gamma$ becomes negative.

Note that we did not plot the analytical growth rates for strongly damped waves. If $\gamma$ is too large, the critical layer, $\left|r-r_{*}\right|<c l_{\gamma}$, covers a sizable fraction of the vortex. This contradicts a key assumption of our analysis.

\section{Breakdown of linear theory}

The above linear theory suggests that a sufficiently large PV gradient in the critical layer inhibits radiation from a VR wave, regardless of the wave amplitude. In reality, the wave amplitude must fall below a finite threshold. In what follows, we will estimate this threshold's upper bound. We will do so with a basic model of critical layer flow that is useful for introducing concepts and making "back of the envelope" calculations. It is not our intention to present a rigorous theory for the nonlinear interaction of a VR wave and its critical layer (cf. Balmforth et al. 2001; Shukhman 1991). Our simplified analysis seems adequate to explain the principal simulation results of section 6 .

\section{a. Basic model of critical layer flow}

Suppose that a single exponentially damped VR wave propagates around a monotonic cyclone. Let us consider the flow near the critical radius $r_{*}$. In this region, a tracer (Lagrangian parcel) approximately obeys Hamiltonian dynamics.

It is convenient to write the Hamiltonian for a tracer in special dimensionless variables. First, we define the initial "bounce frequency,"

$$
\Omega_{b} \equiv\left|2 n a(0) U\left(r_{*}\right) \frac{d \bar{\Omega}}{d r_{*}}\right|^{1 / 2},
$$

and "trapping width,"

$$
l_{\text {trap }}=\frac{2 \Omega_{b}}{\left|n d \bar{\Omega} / d r_{*}\right|} .
$$

Our nomenclature will be justified shortly. Next, we define a dimensionless time variable,

$$
\tau \equiv \Omega_{b} t
$$

In a reference frame that corotates with the VR wave, the approximate time-dependent Hamiltonian of a tracer is 


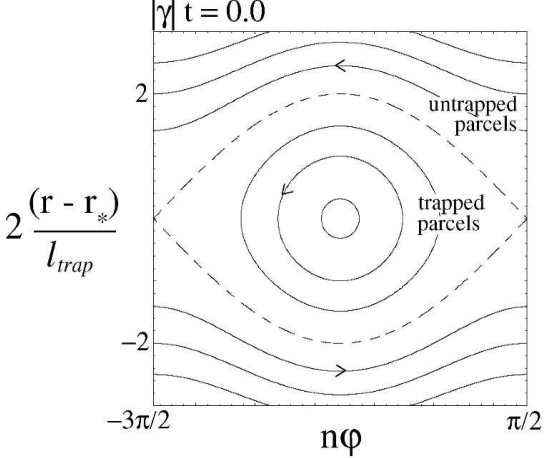

$\mathrm{n} \varphi$

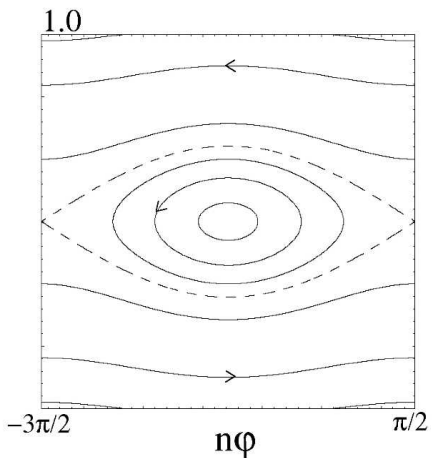

(a)

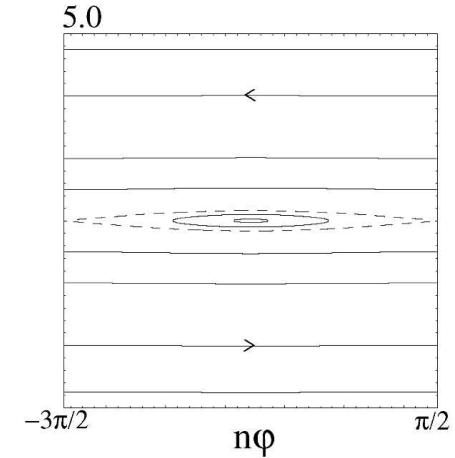

$\mathrm{n} \varphi$ (b)

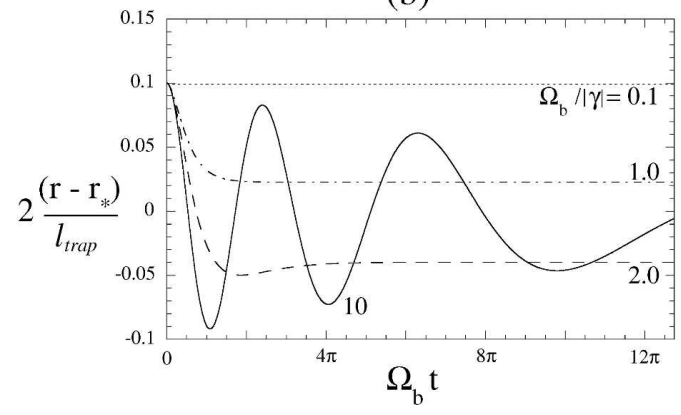

(c)

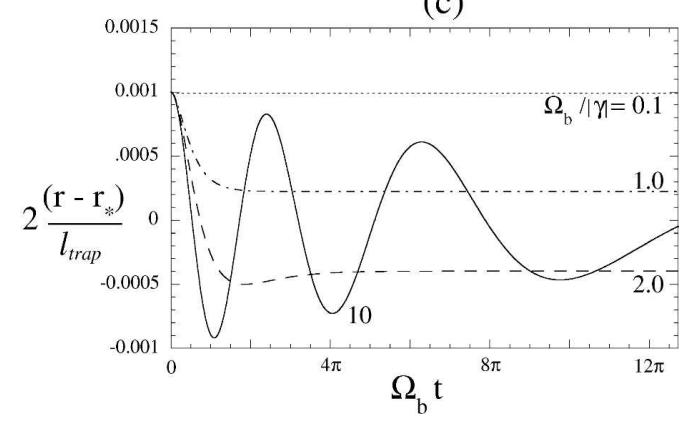

FIG. 7. Basic critical layer dynamics. (a) Three snapshots of the instantaneous flow in the critical layer of a damped VR wave. The reference frame corotates with the wave. (b), (c) Radial motions of two fluid parcels placed near the central fixed point of the trapping region. Different curves correspond to different values of the initial wave amplitude $\left(\Omega_{b} /|\gamma|\right)$.

$$
H=-\frac{p^{2}}{2}-e^{\gamma \tau / \Omega_{b}} \sin (\mu) .
$$

Here $\mu$ and $p$ are the canonically conjugate coordinate and momentum, defined by

$$
\mu \equiv n \varphi, \quad p \equiv 2 \frac{r-r_{*}}{l_{\text {trap }}} .
$$

The equations of motion are ${ }^{6}$

$$
\begin{aligned}
& \frac{d \mu}{d \tau}=\frac{\partial H}{\partial p}=-p, \\
& \frac{d p}{d \tau}=-\frac{\partial H}{\partial \mu}=\cos (\mu) e^{\gamma \tau / \Omega_{b}} .
\end{aligned}
$$

Formally, this approximation is accurate only if $l_{\text {trap }} / r_{*}$ $\ll p \ll l_{v} / l_{\text {trap }}$ in which $l_{v}$ is the radial length scale of the background shear flow or of the VR wave (whichever is smallest). In short, the wave amplitude must be small. In addition, we have temporarily ignored nonlinear

\footnotetext{
${ }^{6}$ Equations (69) are analogous to those of a nonlinear pendulum with a time-dependent gravity (or length) parameter.
}

changes to the mean shear flow and VR wave that might emerge with time.

\section{b. The effect of $V R$ wave amplitude on the nature of critical layer flow}

Figure 7a shows three snapshots of the critical layer flow as it evolves with time. The streamlines correspond to contours of constant $H$. A separatrix (dashed curve) instantaneously divides the flow into regions of trapped and untrapped fluid. The maximum radial halfwidth of the trapping region is $l_{\text {trap }} e^{\gamma \tau / 2 \Omega_{b}}$. Since $\gamma$ is negative, the trapping region thins exponentially with time. Near the center of the trapping region, a tracer has a quasi-elliptical orbit with instantaneous frequency $\Omega_{b} e^{\gamma \tau / 2 \Omega_{b}}$.

Figures $7 \mathrm{~b}$ and $7 \mathrm{c}$ show the radial motions of two tracers, for increasing values of the frequency ratio, $\Omega_{b} /$ $|\gamma|$. Both tracers are placed near the center of the trapping region at $t=0$. Specifically, the initial coordinates and momenta are $(\mu, p)=(-\pi / 2,0.1)$ and $(-\pi / 2$, $0.001)$. Both plots are nearly identical.

If the VR wave is very weak, that is, if $\Omega_{b} /|\gamma| \ll 1$, the tracers complete only a small fraction of their orbits before the separatrix shrinks beneath them. As a result, 
the tracers become untrapped and maintain near constant radial positions. If the $\mathrm{VR}$ wave is very strong, that is, if $\Omega_{b} /|\gamma| \gg 1$, the tracers complete many orbital cycles.

Since PV follows tracer trajectories, its distribution in the critical layer would overturn in the case of a strong VR wave. Because the rate of overturning varies between the center of the trapping region and the separatrix, $\bar{q}$ (viewed as an azimuthal mean) should approximately flatten in the vicinity of $r_{*}$. Linear dynamics does not account for this change. Nonetheless, the linear growth rate formula [Eq. (63)] suggests that zeroing $d \bar{q} / d r_{*}$ would enable IG wave radiation to pump the VR wave without resistance. So, by quasi-linear reasoning, a strong VR wave and its radiation field should ultimately amplify.

In light of the preceding discussion, we propose the following: if

$$
\Omega_{b} /|\gamma| \gtrsim 1,
$$

the VR wave and its radiation field should intensify, even if initially damped. Notably, condition (70) is equivalent to $l_{\text {trap }} \gtrsim 2 l_{\gamma}$.

\section{Nonlinear simulations}

The main purpose of this section is to verify, by numerical experiments, that condition (70) suffices to cause PSR that does not occur in linear theory. In addition, we will analyze in detail the flow of wave activity during the nonlinear evolution of a radiative VR wave. At the end of this section, we will demonstrate that sustained radiation can lead to vortex fission if unimpeded over many vortex rotation periods. Discussion of the latter topic is kept brief because of its close relation to previous numerical studies of acoustic radiation from a compressible vortex (e.g., Chan et al. 1993).

\section{a. The numerical model}

Our numerical integration of the shallow-water equations (8) and (9) is based on the enstrophy-conserving staggered grid model of Sadourny (1975). The simulation domain is a square box with doubly periodic boundary conditions. At large radii, a circular sponge ring absorbs outward-propagating IG waves and keeps the fluid near rest. In addition, $\nabla^{4}$ hyperdiffusion dissipates grid-scale fluctuations to ensure numerical stability.

As shown in Fig. 3, a vortex-scale deformation of a shallow-water cyclone tends to generate longwave radiation in the environment. In our simulations, $l_{\text {rad }}$ is typically 10 times greater than the vortex scale. In contrast, PV stirring in the critical layer generates finescale filaments. Over the course of our simulations, these filaments can thin to a small fraction of the vortex scale. Because the dynamical length scales differ considerably in the cyclone and in the environment, we introduced nested inner and outer grids. The spatial resolution of the inner grid is 6 times greater than that of the outer grid.

For those interested, appendix $\mathrm{C}$ tabulates all of the numerical parameters for the simulations that appear in this paper.

\section{b. Balanced initial conditions}

To initiate radiation from an equilibrated cyclone, we must perturb it. As before, let $\bar{q}(r)$ denote the unperturbed PV profile. In our numerical simulations, we let

$$
q\left(r, \varphi, t=0^{+}\right)=\bar{q}\left(\frac{r}{\sqrt{1-d^{2} / 2}+d \cos (2 \varphi)}\right),
$$

in which $d^{2}<2 / 3$. If $d$ is relatively small, this initial perturbation corresponds to an elliptical deformation. At larger $d$ the deformed vortex resembles a peanut shell. By construction, the deformation conserves the area that is enclosed by each PV contour.

Because there are three prognostic variables (the vector $\mathbf{u}$ and the scalar $\phi$ ), specifying the PV distribution alone does not determine the initial condition. We also require that the flow is balanced. We here define a balanced state to be one in which

$$
\boldsymbol{\nabla} \cdot \mathbf{u}=0
$$

and

$$
\partial_{t} \boldsymbol{\nabla} \cdot \mathbf{u}=0 .
$$

Appendix D explains how we solve Eqs. (71)-(73) for the initial values of the prognostic variables $\mathbf{u}$ and $\phi$.

\section{c. Quasimode revival}

For all cases that we will consider, the above deformation primarily excites a single radiative VR wave. To elaborate, let us expand the radial velocity field in an azimuthal Fourier series,

$$
u=u_{0}(r, t)+\sum_{n=1}^{+\infty}\left[u_{n}(r, t) e^{i n \varphi}+\text { c.c. }\right] \text {. }
$$

In our simulations, the $n=2$ term dominates the expansion. Moreover,

$$
u_{2}(r, t) \approx a(t) e^{-i \omega_{R} t} U(r)
$$

in which $U$ and $\omega_{R}$ are the linear wavefunction and oscillation frequency of the $n=2$ radiative VR wave. 


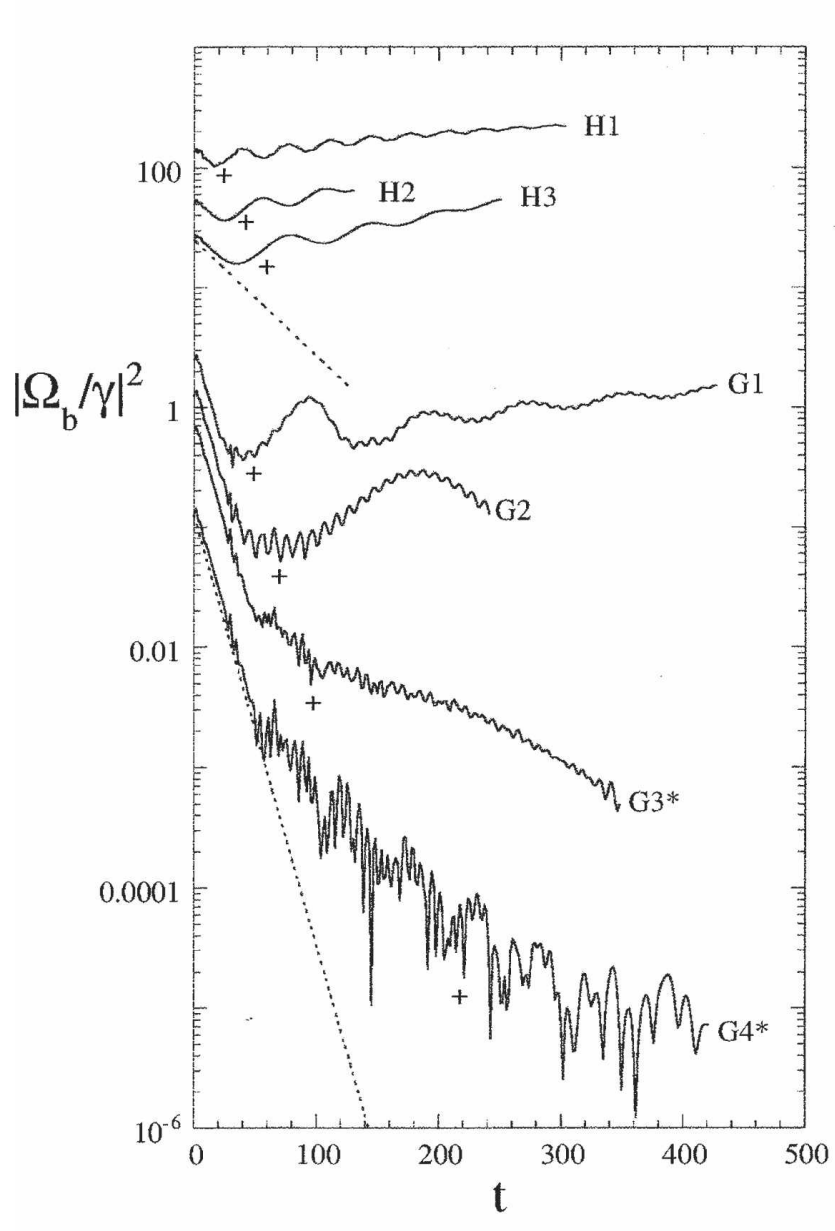

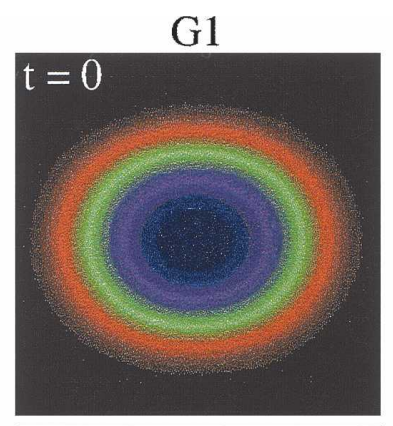
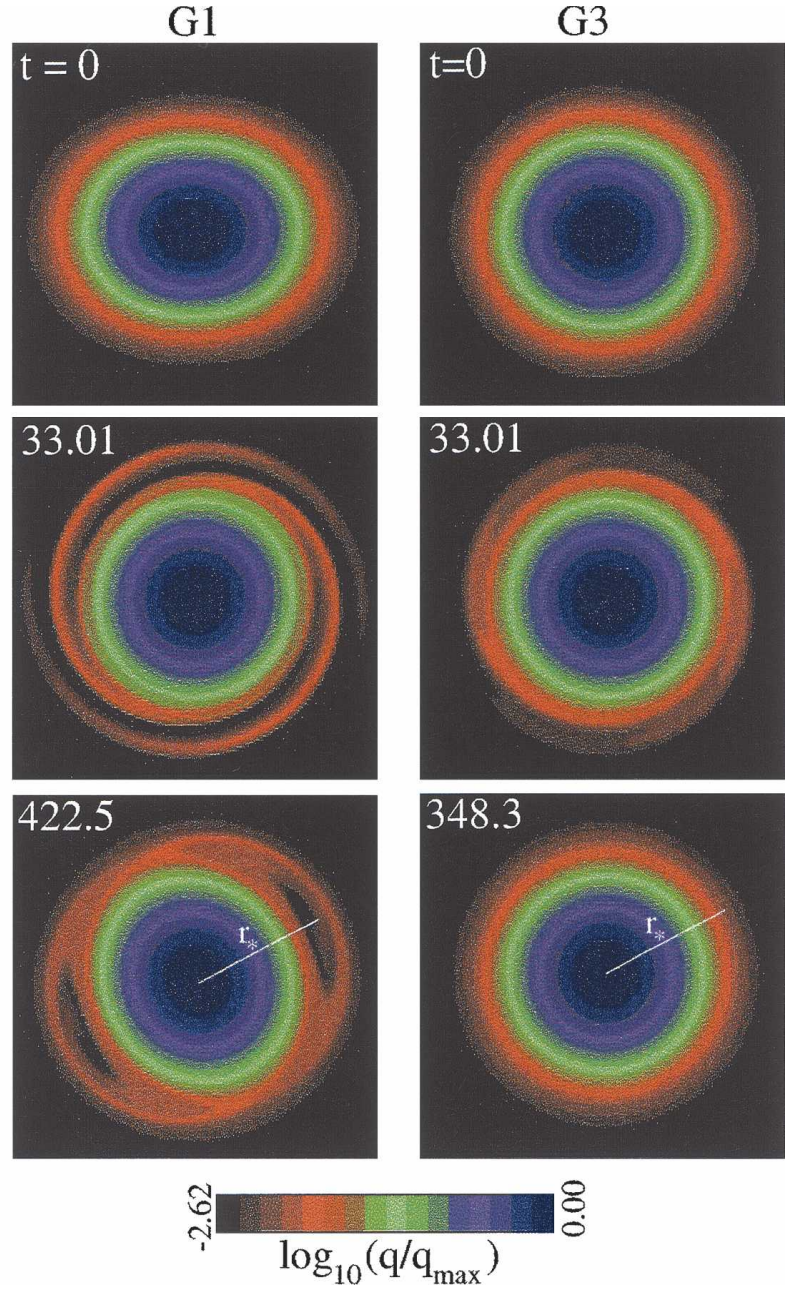

FIG. 8. Nonlinear evolution of linearly damped, radiative VR waves. (left) Wave amplitude, measured by the ratio $\left|\Omega_{b} / \gamma\right|^{2}$, vs time. The dashed curves indicate the early exponential decay that linear theory predicts. The cross-hair beneath each simulation curve indicates the initial bounce period, $t_{b}=2 \pi / \Omega_{b}(0)$, for that simulation. The asterisks indicate filtered data (see appendix E). (right) Potential vorticity vs time for simulations G1 and G3. See Tables 1 and $\mathrm{C} 1$ for simulation details. Time $t$ is in natural units.

The time dependence of $a$ does not necessarily follow linear theory. Nonetheless, its phase varies on a much slower time scale than $\omega_{R}^{-1}$.

For comparison to theory, we will measure the intensity of an excited VR wave by its dynamic bounce frequency,

$$
\Omega_{b}(t) \equiv\left|4 \alpha u_{2}\left(r_{p}, t\right) \frac{d \bar{\Omega}}{d r_{*}}\right|^{1 / 2} .
$$

Here $r_{p}=1.03 r_{o}$ is a radius near the peak of $u_{2}, r_{*}$ is the linear critical radius, and $\alpha \equiv\left|U\left(r_{*}\right) / U\left(r_{p}\right)\right|$. The measured quantity $\Omega_{b}(t)$ closely approximates the instantaneous critical layer mixing rate, insofar as Eq. (75) is accurate. Under the same assumption, its initial value equals the static bounce frequency [Eq. (64)] of section 5 .
Figure 8 plots wave intensity $\left(\Omega_{b}^{2}\right)$ versus time for excited VR waves on both Gaussian and hyperbolic cyclones. Table 1 fully describes the cyclone and wave parameters. Notably, each wave is a quasimode; that is, its linear growth rate $\gamma$ is negative.

Early on, the simulated VR waves decay exponentially with time, in accord with linear theory. Two distinct behaviors occur at later times depending of the value of $\Omega_{b}(0) /|\gamma|$. If this ratio exceeds unity, the VR wave intensifies after a bounce period, $2 \pi / \Omega_{b}(0)$. If this ratio is less than unity, as in curves G3 and G4, the VR wave continues to decay. ${ }^{7}$ This dichotomy was anticipated from the basic considerations of section 5 .

\footnotetext{
${ }^{7}$ Curves G3 and G4 were filtered at late times to remove lowfrequency noise. Appendix E describes the filtering technique.
} 
TABLE 1. Physical simulation parameters: G1-H3 correspond to the simulations in Figs. 8-13 and 15, and H4 corresponds to the top simulation in Fig. 3. All dimensional parameters are in natural units. $\Omega_{b}$ and $l_{\text {trap }}$ are evaluated at $t=0$.

\begin{tabular}{|c|c|c|c|c|c|c|c|c|c|c|c|c|}
\hline Simulation & Cyclone type & Ro & $\mathrm{Fr}^{2}$ & $r_{b}$ & $\omega_{R}$ & $\gamma$ & $r_{*}$ & $l_{\mathrm{rad}}$ & $l_{\gamma}$ & $d$ & $\Omega_{b}$ & $l_{\text {trap }}$ \\
\hline Gl & Gaussian & 40.0 & 0.50 & 22.0 & 0.60 & -0.082 & 2.36 & 14.9 & 0.17 & 0.100 & 0.13 & 0.52 \\
\hline $\mathrm{G} 2$ & Gaussian & 40.0 & 0.50 & 22.0 & 0.60 & -0.082 & 2.36 & 14.9 & 0.17 & 0.050 & 0.09 & 0.37 \\
\hline G3 & Gaussian & 40.0 & 0.50 & 22.0 & 0.60 & -0.082 & 2.36 & 14.9 & 0.17 & 0.025 & 0.065 & 0.26 \\
\hline G4 & Gaussian & 40.0 & 0.50 & 22.0 & 0.60 & -0.082 & 2.36 & 14.9 & 0.17 & 0.005 & 0.03 & 0.12 \\
\hline $\mathrm{H} 1$ & Hyperbolic $(\Delta=0.3)$ & 42.07 & 0.53 & 22.0 & 0.78 & -0.022 & 1.94 & 11.0 & 0.03 & 0.3000 & 0.26 & 0.65 \\
\hline $\mathrm{H} 2$ & Hyperbolic $(\Delta=0.3)$ & 42.07 & 0.53 & 22.0 & 0.78 & -0.022 & 1.94 & 11.0 & 0.03 & 0.1000 & 0.16 & 0.39 \\
\hline $\mathrm{H} 3$ & Hyperbolic $(\Delta=0.3)$ & 42.07 & 0.53 & 22.0 & 0.78 & -0.022 & 1.94 & 11.0 & 0.03 & 0.0500 & 0.11 & 0.28 \\
\hline $\mathrm{H} 4$ & Hyperbolic $(\Delta=0.1)$ & 50.34 & 0.74 & 22.0 & 0.80 & +0.007 & 1.67 & 9.1 & 0.01 & 0.005 & 0.03 & 0.07 \\
\hline
\end{tabular}

\section{d. Nonlinear critical layer stirring}

The two columns on the right-hand side of Fig. 8 show the evolutions of deformed Gaussian cyclones. Different colors represent different levels of PV. In one case, G1, the deformation excites a strong VR wave; that is, $\Omega_{b}(0) /|\gamma|>1$. In the other case, G3, the deformation excites a weak VR wave; that is, $\Omega_{b}(0) /|\gamma|<1$. According to these simulations, PV coils in the critical layer of a strong VR wave, but not in the critical layer of a weak VR wave. Figure 9 provides a closer look at the critical layer flows and leads us to the same conclusion.

Figure 10 shows the initial and late-time profiles of azimuthally averaged $\mathrm{PV}\langle q\rangle_{\varphi}$ in the critical layers of a strong VR wave (G1) and of two weaker VR waves (G3 and G4). The strong wave levels the PV profile, whereas the weaker waves do not. Apparently, the weakest wave $(\mathrm{G} 4)$ has a negligible effect. In section 5 , we argued that leveling the critical layer is what enables radiative pumping to act unopposed. The growth of strong waves but not of weak waves, in Fig. 8, adds merit to this claim.

\section{e. Wave activity analysis}

A wave activity analysis further elucidates the nature of the nonlinear radiative instability. The solid curve in Fig. 11a traces the temporal change of bulk VR wave activity,

$$
\delta A_{b}(t) \equiv A_{b}(t)-A_{b}(0)
$$

in a typical supercritical simulation (H3). For this computation, we let $\delta r_{*}=\sqrt{2} l_{\text {trap }}(0)$ and $r_{v}=r_{*}+$ $1.47 \delta r_{*}$. The dashed curve is the time integral of the right-hand side of Eq. (46). Good agreement between the solid and dashed curves verifies that the simulation
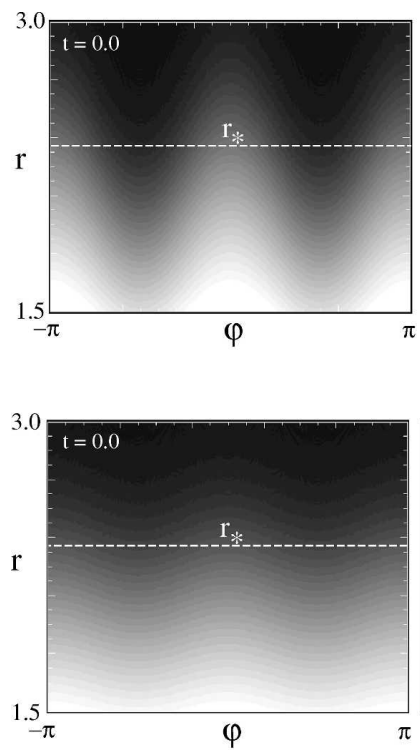

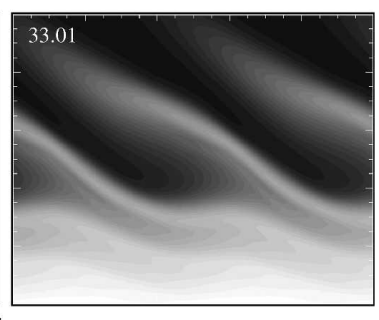

(a)
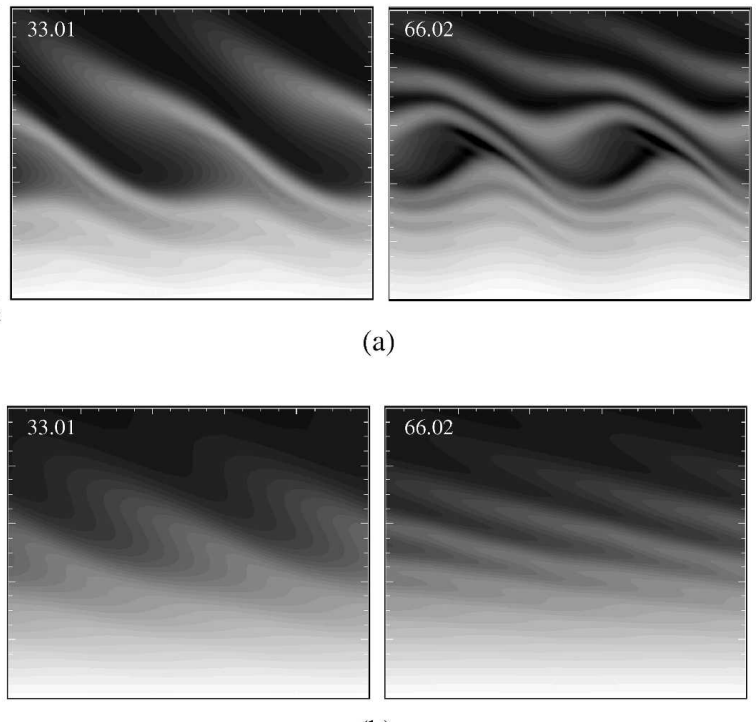
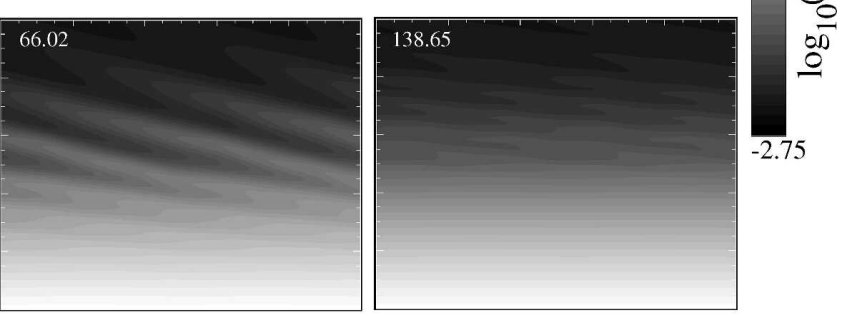

(b)

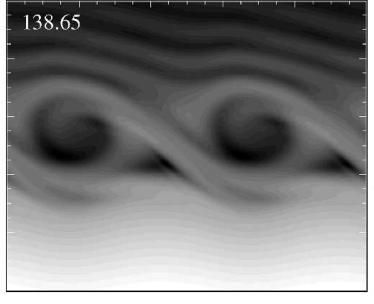

$-0.933$

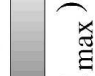

FIG. 9. Potential vorticity stirring in the critical layer. (a) Simulation G1, $\Omega_{b}(0) /|\gamma|>1$. (b) Simulation G3, $\Omega_{b}(0) /|\gamma|<1$. Times and lengths are in natural units. 


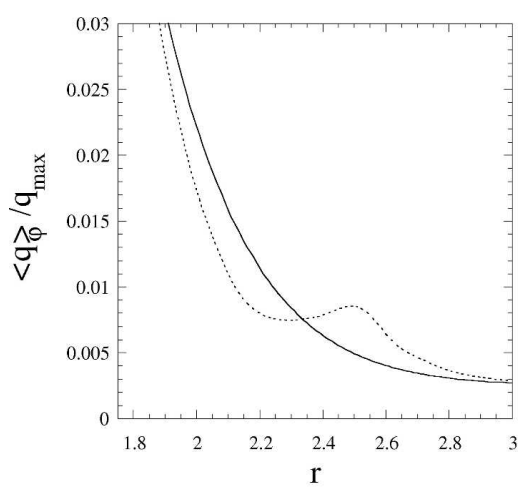

(a)

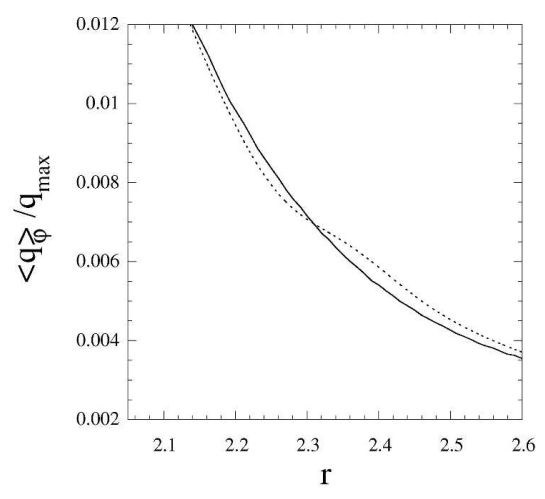

(b)

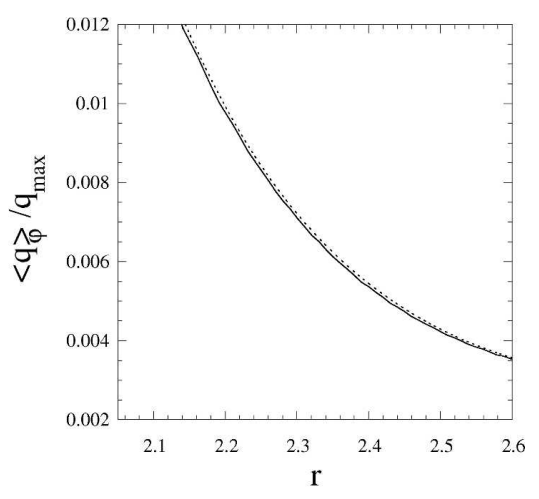

(c)

FIG. 10. Nonlinear changes to the azimuthally averaged potential vorticity in the critical layer, centered at $r_{*}=2.36$. (a) Simulation G1, $\Omega_{b}(0) /|\gamma|=1.58$. (b) Simulation G3, $\Omega_{b}(0) /|\gamma|=0.79$. (c) Simulation G4, $\Omega_{b}(0) /|\gamma|=0.35$. The solid curves represent the initial conditions, whereas the dashed curves correspond to late-time averages in the intervals (a) $138.7<t<427.5$, (b) $138.7<t<345.0$, and (c) $138.7<t<420.9$.

properly evolves wave activity in the bulk of the cyclone.

The top dotted curve is the gain of bulk VR wave activity by IG wave radiation, that is, the time integral of $S\left(r_{v}, t\right)$. The bottom dotted curve is the loss of bulk VR wave activity by critical layer absorption, that is, minus the time integral of the right-hand side of Eq. (49). Initially, critical layer damping is more intense than radiative pumping, and the bulk wave activity decays. However, as the cat's eyes coil PV, the critical layer periodically returns some positive wave activity. This reduces the efficacy of critical layer damping. On the other hand, radiative pumping persists and causes the bulk wave activity to grow.

Note that, overall, the nonlinear critical layer continues to gain wave activity at the expense of the bulk VR wave. There are at least two plausible explanations. To begin with, as the cat's eyes grow, they entrain and stir exterior PV. This seems likely to produce a net gain of critical layer wave activity. In addition, the inner region of our fixed annular critical layer happens to include part of the core PV distribution. So, as the core becomes increasingly elliptical, it should add some wave activity to the critical layer.
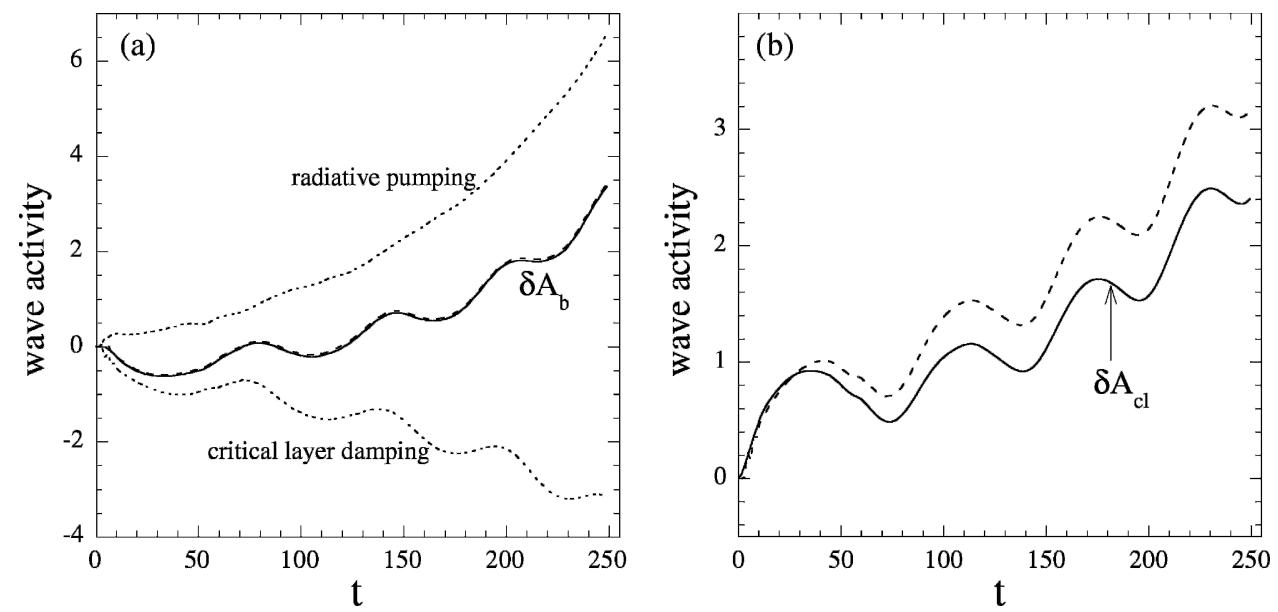

FIG. 11. A closer look at the nonlinear evolution of a supercritical VR wave (simulation H3). (a) The undulatory growth of bulk wave activity (solid curve). The theoretical growth (dashed curve) is the gain by radiative pumping plus the loss by critical layer damping (dotted curves). (b) The growth of critical layer wave activity. By the end of the simulation, the measured value of $\delta A_{\mathrm{cl}}$ (solid curve) is $30 \%$ less than the influx of wave activity into the critical layer (dashed curve). Hyperdiffusion is the likely cause. The wave activities in (a) and (b) are divided by the initial (positive) value of $A_{b}$. Time $t$ is in natural units. 

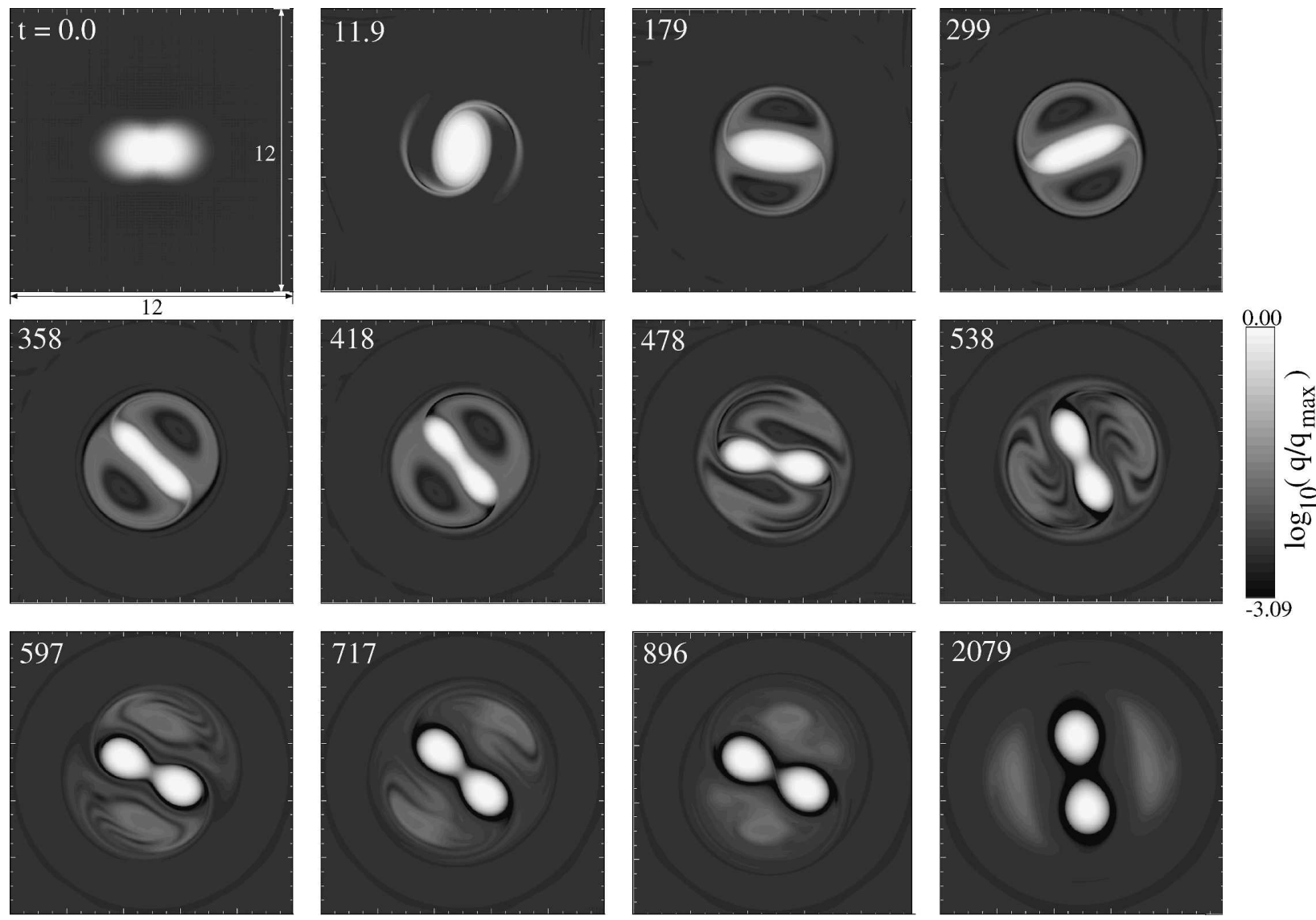

FIG. 12. Fission of a cyclone, induced by IG wave radiation. Time $t$ is in natural units, so $t=2 \pi$ corresponds to one vortex rotation period.

The above simulations, of course, have some degree of numerical error. Although we do not expect this error to cause qualitative inaccuracies, it is necessary to address. To this end, Fig. 11b compares the growth of critical layer wave activity,

$$
\delta A_{\mathrm{cl}}(t) \equiv A_{\mathrm{cl}}(t)-A_{\mathrm{cl}}(0),
$$

to the influx of wave activity from the bulk of the vortex, that is, the time integral of the right-hand side of Eq. (49). By the end of the simulation, the value of $\delta A_{\mathrm{cl}}$ (solid curve) is $30 \%$ less than the influx (dashed curve). The likely cause of dissipation is hyperdiffusion in the numerical model. Hyperdiffusion is enhanced in the critical layer, where nonlinear stirring generates large PV gradients.

\section{f. Vortex fission}

To complete our discussion, we briefly examine the long-term effect of nonlinear PSR. Figure 12 illustrates the evolution of an elliptically deformed hyperbolic cyclone that continuously radiates IG waves. The simula- tion is identical to H1, but with half the grid resolution. Initially, the ellipticity of the core PV distribution decays. From Fig. 8, we know that the decay rate agrees with linear theory. Shortly, the PV turns over in the critical layer, and the core ellipticity starts to grow. Simultaneously, the cat's eyes expand radially, and siphon potential vorticity from the core. Ultimately, the cyclone splits in two. The splitting is consistent with a simplified theory by Ford (1994b) and is analogous simulations of a $2 \mathrm{D}$ compressible vortex by Chan et al. (1993).

Figure 13 shows the evolution of vortex energy, defined by

$$
E_{v} \equiv \mathrm{KE}_{v}+\mathrm{APE}_{v}
$$

in which $\mathrm{KE}_{v}$ and $\mathrm{APE}_{v}$ denote the kinetic energy and the available potential energy of the vortex. Specifically,

$$
\mathrm{KE}_{v} \equiv \int_{0}^{2 \pi} \int_{0}^{r_{v}} d \varphi d r r \frac{\phi \mathbf{u}^{2}}{2}
$$




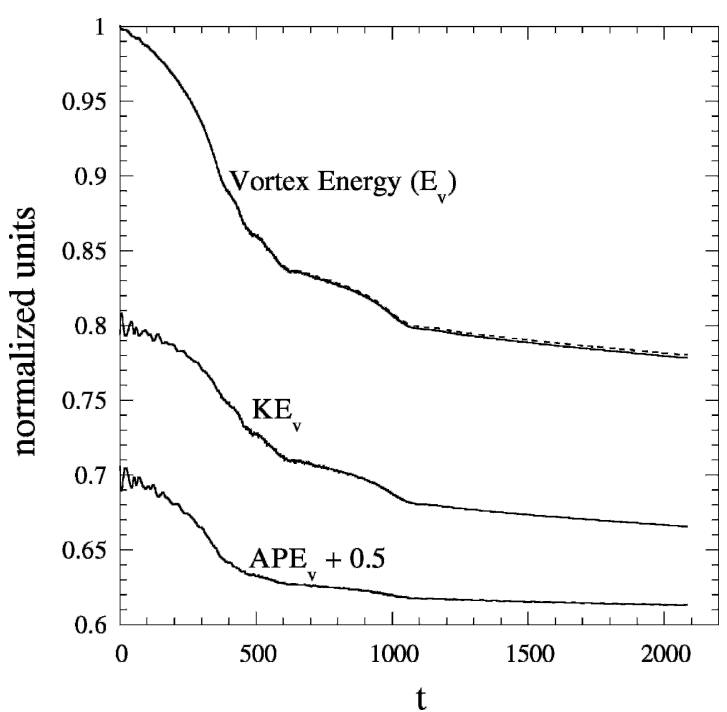

FIG. 13. Temporal decay of vortex energy due to radiation. The solid curves are total, kinetic, and available potential energy integrals, inside the vortex radius $r_{v}$. The dashed curve is the initial total energy minus the energy carried away by IG waves. All energies are normalized to the initial value of total energy.

and

$$
\mathrm{APE}_{v} \equiv \int_{0}^{2 \pi} \int_{0}^{r_{v}} d \varphi d r r \frac{(\phi-\langle\phi\rangle)^{2}}{2}
$$

in which $\langle\phi\rangle$ is the average (conserved) geopotential of the entire simulation domain. The vortex radius is somewhat arbitrary; here we happen to let $r_{v}=6 r_{o}$.

In the absence of dissipation, the vortex energy satisfies the equation

$$
\frac{d E_{v}}{d t}=-\left.\int_{0}^{2 \pi} d \varphi \hat{r} \cdot \mathbf{u}\left(\frac{\phi \mathbf{u}^{2}}{2}+\phi^{2}-\phi\langle\phi\rangle\right)\right|_{r=r_{v}} .
$$

The dashed curve in Fig. 13 is the initial value of $E_{v}$ plus the time integral of the right-hand side of Eq. (82). Good agreement with $E_{v}(t)$ suggests that the energy loss is primarily due to IG wave radiation, as opposed to artificial dissipation. Nonetheless, details of the fission process might change with better resolution of the critical layer.

\section{Concluding remarks}

In this paper, we examined the pure spontaneous radiation (PSR) of IG waves from a monotonic shallow-water cyclone, with Ro greater than unity. Specifically, we considered radiation that is generated by a discrete VR wave in the vortex core. We verified that the radiation has positive feedback and can split the vortex if left unchecked. However, stirring of PV in the critical layer reduces the growth rate of the instability, and can even quench it. Quenching occurs only if

(i) the initial magnitude of the negative PV gradient, $d \bar{q} / d r$, is sufficiently large at $r_{*}$ so that linear critical layer damping prevails over linear radiative pumping [see Eq. (63)], and

(ii) the nonlinear mixing rate $\Omega_{b}$ of $\mathrm{PV}$ in the critical layer is less than the linear decay rate $|\gamma|$ of the VR wave.

Conditions (i) and (ii) were derived in sections 4 and 5, respectively. Condition (ii) was verified by numerical experiments in section 6 .

In addition to the above results, we analyzed the flow of conserved wave activity during nonlinear PSR, from a cyclone that is stable in linear theory. Figure 14 is a diagrammatic summary. On the one hand, the resonant disturbance of PV at $r_{*}$ tends to transfer positive wave activity from the VR wave into its critical layer. The result is damping of the VR wave. On the other hand, IG wave radiation involves the ejection of negative wave activity into the environment, compelling the VR wave to grow. As PV coils inside, the critical layer becomes less absorbent and periodically returns some of its wave activity. This enables radiative pumping to dominate.

Details of the cyclonic equilibrium and its perturbation determine whether or not conditions (i) and (ii) are satisfied. A general survey is beyond the scope of this paper, but some qualitative hypotheses are appropriate. Figure 4 indicates that discrete VR waves in smooth cyclones (those closer to Gaussian than square) are likely to satisfy condition (i). Past computational studies further suggest that deforming a smooth cyclone hardly excites discrete VR waves if the intrinsic Rossby

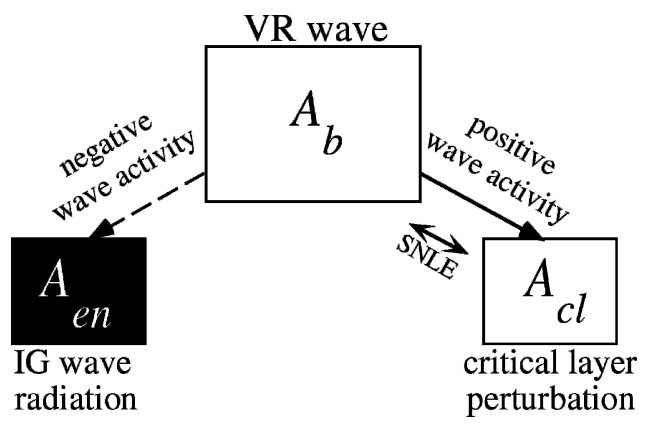

FIG. 14. Flow of wave activity during nonlinear PSR from a monotonic cyclone. Overall, the bulk VR wave ejects negative IG wave activity into the environment and deposits positive wave activity into the critical layer. At later times, the critical layer periodically sends some positive wave activity back to the VR wave. Such transfers are labeled secondary nonlinear exchanges (SNLE) in the diagram. 
deformation radius $L_{R}$ [Eq. (7)] is much less than the radius of maximum wind (e.g., Reasor et al. 2004). In this case, only a very intense impulse might suffice to excite a wave that violates condition (ii).

Section 2 proposed that tropical cyclones, supercell mesocyclones, polar lows, and island wake vortices can spontaneously radiate large-scale spiral IG waves. Such radiation may or may not prove significant in future studies. The contents of this paper may help explain why, but cannot provide the full answer. Atmospheric vortices, of course, are far more complex than inviscid monotonic shallow-water cyclones. They are generally baroclinic and have secondary (poloidal) circulations; in addition, they are often nonmonotonic. Furthermore, their perturbations are influenced by eddy viscosity, boundary layer drag, and moist diabatic processes. One should merely view this paper as another stepping-stone toward a more comprehensive theory of spontaneous radiation from vortical flows in the atmosphere.

Acknowledgments. This work was supported by NSF Grants ATM-0132006, ATM-0349980, ATM-0101781, and ONR Grant N00014-02-1-0474.

\section{APPENDIX A}

\section{Linearized Shallow-Water Model}

The linearized shallow-water equations are obtained from Eqs. (8) and (9) by neglecting quadratic terms in the perturbation fields (marked with primes). The result in a polar coordinate system is given below:

$$
\begin{array}{r}
\left(\partial_{t}+\bar{\Omega} \partial_{\varphi}\right) u^{\prime}-\bar{\xi} v^{\prime}+\partial_{r} \phi^{\prime}=0, \\
\left(\partial_{t}+\bar{\Omega} \partial_{\varphi}\right) v^{\prime}+\bar{\eta} u^{\prime}+\frac{1}{r} \partial_{\varphi} \phi^{\prime}=0, \\
\left(\partial_{t}+\bar{\Omega} \partial_{\varphi}\right) \phi^{\prime}+\frac{1}{r} \partial_{r}\left(r \bar{\phi} u^{\prime}\right)+\frac{\bar{\phi}}{r} \partial_{\varphi} v^{\prime}=0 .
\end{array}
$$

The auxiliary equation for the linearized PV perturbation is

$$
\left(\partial_{t}+\bar{\Omega} \partial_{\varphi}\right) q^{\prime}+\frac{d \bar{q}}{d r} u^{\prime}=0
$$

in which

$$
q^{\prime}=\frac{\partial_{r}\left(r v^{\prime}\right)-\partial_{\varphi} u^{\prime}}{r \bar{\phi}}-\frac{\bar{\eta}}{\bar{\phi}^{2}} \phi^{\prime} .
$$

Note that in linear theory, Eq. (35) defines the angular pseudomomentum $\mathcal{L}$. Equation (36) still governs the evolution of $\mathcal{L}$, but $\mathbf{F}$ reduces to its quadratic approximation.

\section{APPENDIX B}

\section{Polarization Equations}

Sections $3 \mathrm{c}$ and $3 \mathrm{e}$ explained how to calculate the geopotential wavefunction $\Phi$ of an eigenmode or quasimode, respectively. The following equations relate the radial $(U)$ and azimuthal $(V)$ velocity wavefunctions to $\Phi$ :

$$
U=\frac{i}{\bar{\eta} \bar{\xi}-\sigma^{2}}\left(\sigma \frac{d \Phi}{d r}-\frac{n \bar{\xi}}{r} \Phi\right)
$$

and

$$
V=\frac{1}{\bar{\eta} \bar{\xi}-\sigma^{2}}\left(\bar{\eta} \frac{d \Phi}{d r}-\frac{n \sigma}{r} \Phi\right) .
$$

In addition, the PV wavefunction satisfies

$$
Q=-\frac{i}{\sigma} \frac{d \bar{q}}{d r} U
$$

Equations (B1)-(B3) are derived by substituting the traveling wave ansatz [Eq. (20) and its analogues] into the linearized shallow-water equations [(A1)-(A4)].

\section{APPENDIX C}

\section{Numerical Parameters}

Table $\mathrm{C} 1$ lists the numerical parameters, defined below, that were used for all simulations $(\mathrm{G} 1-\mathrm{H} 4)$ in this paper. Simulation H1-lr corresponds to the lowresolution vortex fission experiment. It has the same physical parameters as H1 (see Table 1).

\section{a. Discretization parameters}

The numerical model integrates the shallow-water equations on two concentric square grids. ${ }^{8}$ The inner fine grid has spacing $\delta x$ and the outer coarse grid has spacing $\Delta X$. Each side of the fine grid has length $L_{\mathrm{fg}}$, and each side of the coarse grid has length $L_{\mathrm{cg}}$. The flow is evolved forward in time using a fourth-order RungeKutta scheme, in increments of size $\delta t$.

\section{b. Artificial damping parameters}

The numerical model adds two dissipation terms,

$$
\mathcal{H D}+\mathcal{S P}
$$

\footnotetext{
${ }^{8}$ With staggering, there are actually eight total grids.
} 
TABLE C1. Numerical simulation parameters, in natural units.

\begin{tabular}{cccccccccc}
\hline \hline Simulation & $\nu_{\mathrm{fg}} \times 10^{7}$ & $r_{\text {spng }}$ & $\delta r_{\text {spng }}$ & $\beta$ & $L_{\mathrm{cg}}$ & $L_{\mathrm{fg}}$ & $\delta x$ & $\Delta X$ & $\delta t \times 10^{2}$ \\
\hline G1 & 0.061 & 40 & 2 & 3.8 & 96 & 12.1 & 0.01 & 0.06 & 0.69 \\
G2 & 0.061 & 40 & 2 & 3.8 & 96 & 12.1 & 0.01 & 0.06 & 0.66 \\
G3 & 0.061 & 40 & 2 & 3.8 & 96 & 12.1 & 0.01 & 0.06 & 0.69 \\
G4 & 0.061 & 40 & 2 & 3.8 & 96 & 12.1 & 0.01 & 0.06 & 0.69 \\
H1-lr & 1.071 & 40 & 2 & 4.2 & 96 & 12.2 & 0.02 & 0.12 & 1.42 \\
H1 & 0.067 & 40 & 2 & 4.2 & 96 & 12.1 & 0.01 & 0.06 & 0.71 \\
H2 & 0.067 & 40 & 2 & 4.2 & 96 & 12.1 & 0.01 & 0.06 & 0.71 \\
H3 & 0.067 & 40 & 2 & 4.2 & 96 & 12.1 & 0.01 & 0.06 & 0.71 \\
H4 & 0.063 & 40 & 2 & 3.9 & 96 & 12.1 & 0.01 & 0.06 & 0.80 \\
\hline
\end{tabular}

to the right-hand sides of the momentum and continuity equations [(8) and (9)]. The first term, hyperdiffusion, has the form

$$
\mathcal{H D} \equiv-\nu \nabla^{4} \chi,
$$

in which $\chi$ is either $\mathbf{u}$ or $\phi$ for the momentum or continuity equation, respectively. The discretized version of $\nabla^{4}$ is a second-order finite difference approximation of $\nabla^{2}$ applied twice. The hyperdiffusion coefficient $\nu$ differs for the fine and coarse grids. If $\nu_{\mathrm{fg}}$ is the fine grid coefficient, then $\nu_{\mathrm{cg}}=(\Delta X / \delta x)^{4} \nu_{\mathrm{fg}}$ is the coarse grid coefficient.

The second term is a sponge that absorbs outward propagating IG waves at large radii. For the momentum equation,

$$
S P \equiv-\frac{\beta}{2}\left[1-\tanh \left(\frac{r_{\text {spng }}-r}{\delta r_{\text {spng }}}\right)\right] \mathbf{u} .
$$

The positive constant $\beta$ is the maximal damping rate. The radius $r_{\text {spng }}$ is far from the vortex, where the fluid is initially at rest. In addition, $\delta r_{\text {spng }} \ll r_{\text {spng. }}$. The functional form of $S P$ ensures that the sponge is active (inactive) at radii greater than (less than) $r_{\text {spng. For the }}$ continuity equation, $S P=0$ so that the sponge neither creates nor destroys mass.

\section{APPENDIX D}

\section{Initialization}

The first balance condition [Eq. (72)] is automatically satisfied by expressing the initial velocity field as the cross gradient of a streamfunction; that is,

$$
\mathbf{u} \equiv \hat{\mathbf{z}} \times \nabla \psi
$$

Substituting Eq. (D1) into Eq. (12), we obtain

$$
\nabla^{2} \psi=q \phi-f
$$

in which $q$ is specified by Eq. (71). The second balance condition [Eq. (73)], combined with the divergence of the momentum equation $[(8)]$, further implies that

$$
\nabla^{2} \phi=f \nabla^{2} \psi-2\left(\partial_{x y} \psi\right)^{2}+2 \partial_{x x} \psi \partial_{y y} \psi
$$

Finally, the constraint of zero total circulation can be written

$$
\int d \mathbf{r}^{2}(q \phi-f)=0,
$$

in which the area integral covers the entire domain of the simulation.

The above transformation reduces initialization to the simultaneous solution of two coupled Poisson equations (D2) and (D3), with periodic boundary conditions, and an integral equation [(D4)]. We solve a discretized version of this system for $\psi$ and $\phi$ by iterative relaxation. The initial velocity field is then obtained from Eq. (D1).

\section{APPENDIX E}

\section{Data Filtering}

Two curves in Fig. 8, G3 and G4, are filtered signals of radiative VR waves. Filtering was necessary at late times when the VR wave amplitudes fell beneath the low-frequency noise threshold. The following explains how the filtered signals were obtained from the raw data.

To begin with, Fig. E1 contrasts the filtered and raw signals for both simulations. The top and bottom plots show the amplitude and phase of $u_{2}\left(r_{p}, t\right) \propto \Omega_{b}^{2} e^{i \theta_{u}}$. Let us first consider the raw data. In both cases the exponential decay of the perturbation amplitude halts near $t$ $=80$. Simultaneously, the oscillation frequency, $-d \theta_{u} /$ $d t$, decreases by orders of magnitude. The dashed curves in all plots were obtained from a numerical integration of the linearized shallow-water equations. They accurately describe the dominant component of the raw signal; that is, the abrupt change at $t=80$ is a linear effect.

Evidently, a slow discrete mode appears to overtake the signal of the radiative VR wave. This mode exists because the cyclone is only quasi monotonic: recall from section $3 \mathrm{~b}$ that the PV jumps slightly at the distant 

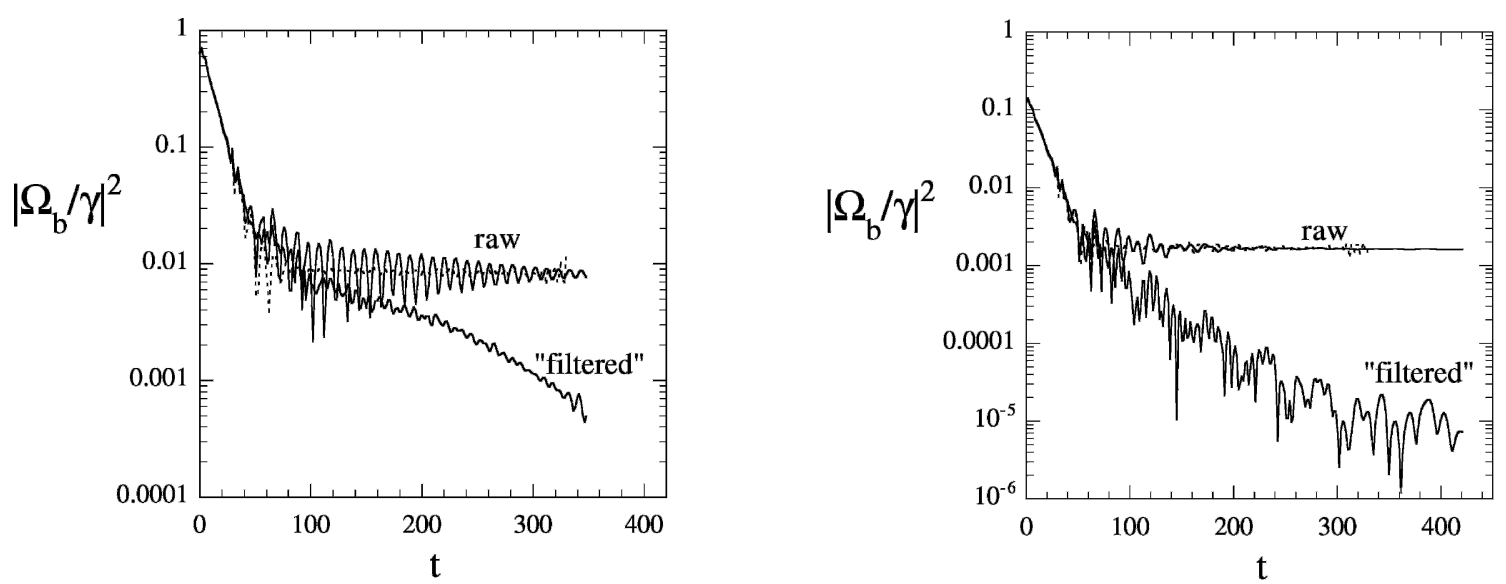

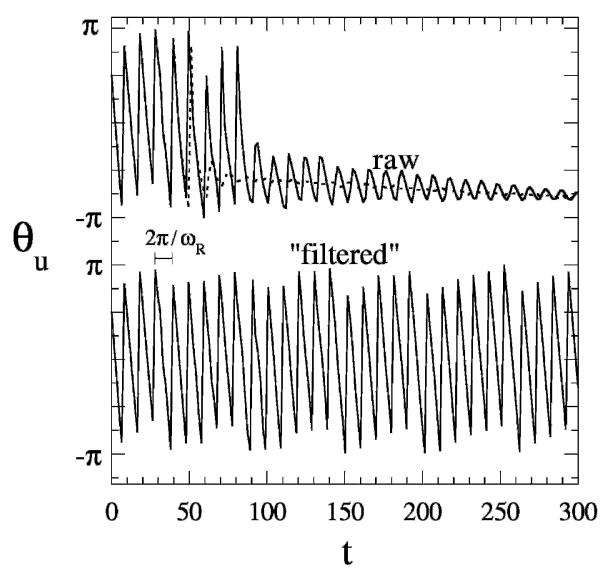

(a)

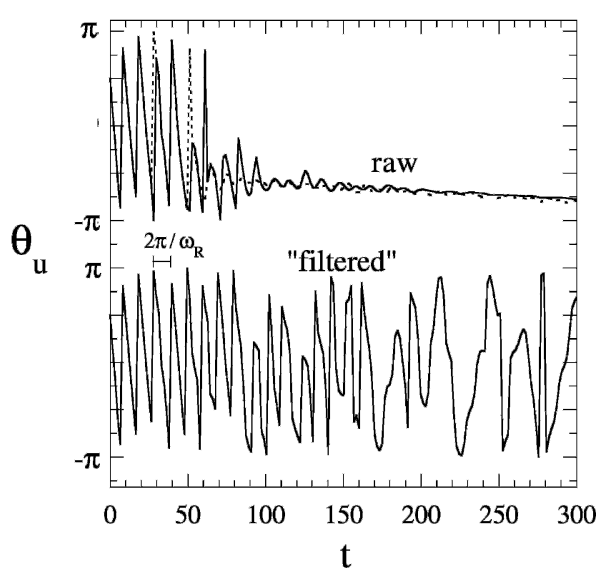

(b)

FIG. E1. Comparison of raw and filtered data for numerical experiments (a) G3 and (b) G4. The solid/dashed curves were obtained from nonlinear/linear simulations. Only nonlinear data were filtered. The data were sampled every 1.7 natural time units. The horizontal bars in the phase plots indicate the linear oscillation period of the $n=2$ radiative VR wave.

radius $r=r_{b}$. The slow mode is peaked at $r_{b}$, but is nonzero in the vortex core, which contains the probe radius $r_{p}$. It does not produce IG wave radiation because its frequency is merely one-tenth of the Coriolis parameter $f$.

The slow mode is of little interest to our discussion. Presumably, it has negligible influence on the much faster radiative mode. That is, it merely adds linearly to the signal. To remove the slow mode from the raw signal we follow a simple procedure. First, we fit the late-time measurements of $u_{2}\left(r_{p}, t\right)$ to a function of the form $a_{\text {slow }} e^{-i \omega_{\text {slow }} t}$. Here $a_{\text {slow }}$ and $\omega_{\text {slow }}$ are complex parameters. We then subtract the fit function from the original $u_{2}\left(r_{p}, t\right)$. We refer to the difference as the filtered signal.

For simulation G3, in which $\Omega_{b}(0) /|\gamma|=0.79$, the filtering leaves a nearly monochromatic signal, corresponding to the radiative VR wave. For simulation G4, in which $\Omega_{b}(0) /|\gamma|=0.35$, the filtered signal is less clean, especially past $t=160$. Beyond this time, we can no longer assert that it represents the original VR wave. Moreover, we can no longer connect its amplitude to the mixing rate of the critical layer. A combination of physical waves and discretization noise likely accounts for the broadened frequency spectrum.

\section{REFERENCES}

Abdullah, A. J., 1966: The "musical" sound emitted by a tornado. Mon. Wea. Rev., 94, 213-220.

Balmforth, N. J., 1999: Shear instability in shallow water. J. Fluid Mech., 387, 97-127.

— S. G. Llewellyn Smith, and W. R. Young, 2001: Disturbing vortices. J. Fluid Mech., 426, 95-133.

Bedard, A. J., 2005: Low-frequency atmospheric acoustic energy associated with vortices produced by thunderstorms. Mon. Wea. Rev., 133, 241-263.

Briggs, R. J., J. D. Daugherty, and R. H. Levy, 1970: Role of Lan- 
dau damping in crossed-field electron beams and inviscid shear flow. Phys. Fluids, 13, 421-432.

Broadbent, E. G., and D. W. Moore, 1979: Acoustic destabilization of vortices. Philos. Trans. Roy. Soc. London A, 290, 353-371.

Chan, W. M., K. Shariff, and T. H. Pulliam, 1993: Instabilities of two-dimensional inviscid compressible vortices. J. Fluid Mech., 253, 173-209.

Chimonas, G., and H. M. Hauser, 1997: The transfer of angular momentum from vortices to gravity swirl waves. J. Atmos. Sci., 54, 1701-1711.

Chow, K. C., and K. L. Chan, 2003: Angular momentum transports by moving spiral waves. J. Atmos. Sci., 60, 2004-2009.

- — — bands in tropical cyclones. J. Atmos. Sci., 59, 2930-2950.

Corngold, N. R., 1995: Linear response of the two-dimensional pure electron plasma: Quasimodes for some model profiles. Phys. Plasmas, 2, 620-628.

Ford, R., 1994a: The instability of an axisymmetric vortex with monotonic potential vorticity in rotating shallow water. $J$. Fluid Mech., 280, 303-334.

- 1994b: The response of a rotating ellipse of uniform potential vorticity to gravity wave radiation. Phys. Fluids, 6, 36943704.

—, M. E. McIntyre, and W. A. Norton, 2000: Balance and the slow quasi-manifold: Some explicit results. J. Atmos. Sci., 57, 1236-1254.

Guinn, T. A., and W. H. Schubert, 1993: Hurricane spiral bands. J. Atmos. Sci., 50, 3380-3403.

Howe, M. S., 2001: Vorticity and the theory of aerodynamic sound. J. Eng. Math., 41, 367-400.

Kambe, T., 1986: Acoustic emissions by vortex motions. J. Fluid Mech., 173, 643-666.

Killworth, P. D., and M. E. McIntyre, 1985: Do Rossby-wave critical layers absorb, reflect, or over-reflect? J. Fluid Mech., 161, 449-492.

Kop'ev, V. F., and E. A. Leont'ev, 1983: Acoustic instability of an axial vortex. Sov. Phys. Acoust., 29, 111-115.

— certain steady-state vortices. Sov. Phys. Acoust., 31, 205-207.

$\ldots$, and — 1988: Acoustic instability of planar vortex flows with circular streamlines. Sov. Phys. Acoust., 34, 276-278.

Kurihara, Y., 1976: On the development of spiral bands in tropical cyclones. J. Atmos. Sci., 33, 940-958.

Li, H., S. A. Colgate, B. Wendroff, and R. Liska, 2001: Rossby wave instability of thin accretion disks. III. Nonlinear simulations. Astrophys. J., 551, 874-896.

Lighthill, M. J., 1952: On sound generated aerodynamically, I. General theory. Proc. Roy. Soc. London, 211A, 564-587.

Lord, K., 1880: On the vibrations of a columnar vortex. Philos. Mag., 10, 155-168.

Maslowe, S. A., 1986: Critical layers in shear flow. Annu. Rev. Fluid. Mech., 18, 405-432.

Métais, O., and J. R. Herring, 1989: Numerical simulations of freely evolving turbulence in stably stratified fluids. J. Fluid Mech., 202, 117-148.

Montgomery, M. T., and L. J. Shapiro, 1995: Generalized Charney-Stern and Fjortoft theorems for rapidly rotating vortices. J. Atmos. Sci., 52, 1829-1833.
—_, and J. Enagonio, 1998: Tropical cyclogenesis via convectively forced vortex Rossby waves in a three-dimensional quasigeostrophic model. J. Atmos. Sci., 55, 3176-3207.

O'Neil, T. M., 1965: Collisionless damping of nonlinear plasma oscillations. Phys. Fluids, 8, 2255-2262.

O'Sullivan, D., and T. J. Dunkerton, 1995: Generation of inertiagravity waves in a simulated life cycle of baroclinic instability. J. Atmos. Sci., 52, 3695-3705.

Papaloizou, J. C. B., and J. E. Pringle, 1987: The dynamic stability of differentially rotating discs-III. Mon. Not. Roy. Astron. Soc., 225, 267-283.

- and D. N. C. Lin, 1995: Theory of accretion disks I: Angular momentum transport processes. Ann. Rev. Astron. Astrophys., 33, 505-540.

Pillai, S., and R. W. Gould, 1994: Damping and trapping in 2D inviscid fluids. Phys. Rev. Lett., 73, 2849-2852.

Plougonven, R., and V. Zeitlin, 2002: Internal gravity wave emission from a pancake vortex: An example of wave-vortex interaction in strongly stratified flows. Phys. Fluids, 14, 12591268.

Polvani, L. M., J. C. McWilliams, M. A. Spall, and R. Ford, 1994: The coherent structures of shallow-water turbulence: Deformation-radius effects, cyclone/anticyclone asymmetry and gravity-wave generation. Chaos, 4, 177-186.

Reasor, P. D., M. T. Montgomery, and L. D. Grasso, 2004: A new look at the problem of tropical cyclones in shear flow: Vortex resiliency. J. Atmos. Sci., 61, 3-22.

Sadourny, R., 1975: The dynamics of finite-difference models of the shallow-water equations. J. Atmos. Sci., 32, 680-689.

Schecter, D. A., and M. T. Montgomery, 2004: Damping and pumping of a vortex Rossby wave in a monotonic cyclone: Critical layer stirring versus inertia-buoyancy wave emission. Phys. Fluids, 16, 1334-1348.

, D. H. E. Dubin, A. C. Cass, C. F. Driscoll, I. M. Lansky, and T. M. O'Neil, 2000: Inviscid damping of asymmetries on a two-dimensional vortex. Phys. Fluids, 12, 2397-2412.

-, M. T. Montgomery, and P. D. Reasor, 2002: A theory for the vertical alignment of a quasigeostrophic vortex. J. Atmos. Sci., 59, 150-168.

Shapiro, L. J., and M. T. Montgomery, 1993: A three-dimensional balance theory for rapidly rotating vortices. J. Atmos. Sci., 50, 3322-3335.

Shukhman, I. G., 1991: Nonlinear evolution of spiral density waves generated by the instability of the shear layer in a rotating compressible fluid. J. Fluid Mech., 233, 587-612.

Sozou, C., 1987: Adiabatic perturbations in an unbounded Rankine vortex. Proc. Roy. Soc. London A, 411, 207-224.

Spencer, R. L., and S. N. Rasband, 1997: Damped diocotron quasi-modes of nonneutral plasmas and inviscid fluids. Phys. Plasmas, 4, 53-60.

Vanneste, J., and I. Yavneh, 2004: Exponentially small inertiagravity waves and the breakdown of quasigeostrophic balance. J. Atmos. Sci., 61, 211-223.

Willoughby, H. E., F. D. Marks Jr., and R. J. Feinberg, 1984: Stationary and moving convective bands in hurricanes. J. Atmos. Sci., 41, 3189-3211.

Zeitlin, V., 1991: On the backreaction of acoustic radiation for distributed two-dimensional vortex structures. Phys. Fluids A, 3, 1677-1680. 